\title{
A NEW ALGORITHM BASED ON FACTORIZATION FOR HETEROGENEOUS DOMAIN DECOMPOSITION
}

\author{
MARTIN J. GANDER* ${ }^{*}$ LAURENCE HALPERN ${ }^{\dagger}$, AND VÉRONIQUE MARTIN ${ }^{\ddagger}$
}

\begin{abstract}
Often computational models are too expensive to be solved in the entire domain of simulation, and a cheaper model would suffice away from the main zone of interest. We present for the concrete example of an evolution problem of advection reaction diffusion type a heterogeneous domain decomposition algorithm which allows us to recover a solution that is very close to the solution of the fully viscous problem, but solves only an inviscid problem in parts of the domain. Our new algorithm is based on the factorization of the underlying differential operator, and we therefore call it factorization algorithm. We give a detailed error analysis, and show that we can obtain approximations in the viscous region which are much closer to the viscous solution in the entire domain of simulation than approximations obtained by other heterogeneous domain decomposition algorithms from the literature.
\end{abstract}

Key words. Heterogeneous domain decomposition, viscous problems with inviscid approximations, transmission conditions, factorization algorithm

AMS subject classifications. 65M55, 65M15

1. Introduction. The coupling of different types of partial differential equations is an active field of research, since the need for such coupling arises in various applications. A first main area is the simulation of complex objects, composed of different materials, which are naturally modeled by different equations; fluid-structure interaction is a typical example, and many techniques have been developed for this type of coupling problems, see for example the book [33, or the review on the immersed boundary method [32, and 9] for domain decomposition coupling techniques. A very important area of application is the simulation of the cardiovascular system [16. A second main area is when homogeneous objects are simulated, but the partial differential equation modeling the object is too expensive to solve over the entire object, and a simpler, less expensive model would suffice in most of the object to reach the desired accuracy; air flow around an airplane is a typical example, where viscous effects are important close to the airplane, but can be neglected further away, see the early publication [10, and also [7] and the references therein. An automatic approach for neglecting the diffusion in parts of the domain is the $\chi$-formulation, see 27] [5], and there are also techniques based on virtual control, originating in [11, see [1] for the case with overlap, and [25] for the case without, and also [12] for virtual control with variational coupling conditions. A third emerging area is the coupling of equations across dimensions, for example the blood flow in the artery can be modeled by a one dimensional model, but in the heart, it needs to be three dimensional, see for example [15. All these techniques have become known in the domain decomposition community under the name heterogeneous domain decomposition methods, a terminology sparked by the review [36], and the literature has become vast in this field.

We are interested in this paper in the second situation, where the motivation

\footnotetext{
*Section de mathématiques, Université de Genève, 2-4 rue du Lièvre, CP 64, CH-1211 Genève 4, Switzerland. Martin.Gander@unige.ch

†LAGA, Université Paris XIII, 99 Avenue J.-B. Clément, 93430 Villetaneuse, France. halpern@math.univ-paris13.fr

${ }^{\ddagger}$ LAMFA UMR-CNRS 7352, Université de Picardie Jules Verne, 33 Rue St. Leu, 80039 Amiens, France. veronique.martin@u-picardie.fr
} 
for using different equations comes from the fact that we would like to use simpler, less expensive equations in areas of the domain where the full model is not needed, and we use as our guiding example the advection reaction diffusion equation. We are in principle interested in the fully viscous solution, but we would like to solve only an advection reaction equation for computational savings in part of the domain. Coupling conditions for this type of problem have been developed in the seminal paper [23], but with the first situation described above in mind, i.e. there is indeed a viscous and an inviscid physical domain, and the coupling conditions are obtained by a limiting process as the viscosity goes to zero, see also [24, and [3, 8] for an innovative correction layer, and [6] for the steady case.

Dubach developed in his PhD thesis [13] coupling conditions based on absorbing boundary conditions, and such conditions have been used in order to define heterogeneous domain decomposition methods in [18. A fundamental question however in the second situation described above is how far the solution obtained from the coupled problem is from the solution of the original, more expensive one on the entire domain. A first comparison of different transmission conditions focusing on this aspect appeared in [19]. In [20, coupling conditions were developed for stationary advection reaction diffusion equations in one spatial dimensions, which lead to solutions of the coupled problem that can be exponentially close to the fully viscous solution, and rigorous error estimates are provided. The coupling conditions are based on the factorization of the differential operator, see also [29], and the exact factorization can be used in this one dimensional steady case. We study in this paper time dependent advection reaction diffusion problems, where the exact factorization of the differential operator can not be used any more, due to the non-local nature of the factors, and new ideas are needed in order to obtain better coupling conditions than the classical ones developed for situation one, where the domains are really physically different.

We present in Section 2 our new factorization algorithm. In Section 3 and 4 we give a detailed error analysis of our algorithm, and prove asymptotic error estimates when the viscosity is becoming small. In Section 5 we present numerical experiments which show that our theoretical error estimates are sharp, and that the new factorization algorithm gives approximate solutions which are one order of magnitude more accurate in the viscous region than the best heterogeneous domain decomposition methods known from the literature.

2. A new coupling algorithm based on factorization. We now explain how the factorization technique that led to coupling conditions of excellent quality for one dimensional problems in [20] can be used to obtain a new coupling algorithm for evolution problems which we will call factorization algorithm.

2.1. Model problem. We consider the time dependent advection reaction diffusion equation

$$
\begin{aligned}
\mathcal{L}_{a d} u:=\partial_{t} u-\nu \partial_{x}^{2} u+a \partial_{x} u+c u & =f & & \text { in }\left(-L_{1}, L_{2}\right) \times(0, T), \\
\mathcal{B}_{1} u\left(-L_{1}, \cdot\right) & =g_{1} & & \text { on }(0, T), \\
\mathcal{B}_{2} u\left(L_{2}, \cdot\right) & =g_{2} & & \text { on }(0, T), \\
u(x, 0) & =h & & \text { in }\left(-L_{1}, L_{2}\right),
\end{aligned}
$$

where $a$ is the velocity field, $\nu>0$ is the viscosity, and $c>0$ is a reaction term. The $\mathcal{B}_{j}, j=1,2$ are suitable boundary operators, representing Dirichlet or absorbing boundary conditions. We consider the following choice of these operators, depending 
on the sign of the advection term $a$,

\begin{tabular}{|c||c|c|}
\hline & $\mathcal{B}_{1}$ & $\mathcal{B}_{2}$ \\
\hline$a>0$ & $I d$ & $\partial_{t}+a \partial_{x}+c$ \\
\hline$a<0$ & $I d$ & $I d$ \\
\hline
\end{tabular}

In the case $a>0$, the flow is given at the inflow boundary, and an absorbing boundary condition is prescribed at the outflow boundary. This can be compared to the situation of the tail of a wing, where the flow goes from the complicated model region into the simplified model region. For negative $a$, the flow is prescribed at the inflow and outflow boundary, which can be compared to the situation of the front of the wing, where the flow goes from the simplified model region into the complicated model region, and a boundary layer forms.

We assume that the initial data $h$ is compactly supported in $\left(-L_{1}, 0\right)$. The forcing term $f$ is compactly supported in $\left(-L_{1}, L_{2}\right) \times(0, T]$, and the boundary values $g_{1}$ and $g_{2}$ are compactly supported in $(0, T]$. Regularity and compatibility conditions on the data need to be enforced to have a sufficiently regular solution, see Section 3 .

2.2. The new algorithm based on factorization. Using Nirenberg's factorization, we can factor the advection-diffusion operator into a product of two evolution operators in opposite $x$ directions,

$$
-\nu \partial_{x}^{2}+a \partial_{x}+c+\partial_{t}=-\nu\left(\partial_{x}+\mathcal{P}_{+}\left(\partial_{t}\right)\right)\left(\partial_{x}+\mathcal{P}_{-}\left(\partial_{t}\right)\right) \quad\left(\bmod \mathcal{C}^{\infty}\right)
$$

This factorization has been used to design absorbing boundary conditions and paraxial equations for hyperbolic problems, see [2]. For parabolic problems, Nataf and coauthors 29, 34 computed approximations of $u$ via a double sweep, and also obtained transmission conditions for Schwarz domain decomposition methods [35], which led to the new class of optimized Schwarz methods, see [17] for an overview. The same factorization can also be used to obtain incomplete LU preconditioners [21, 22, and is the underlying mathematical structure of the recently developed sweeping preconditioner [14. We now use this factorization to define our new factorization algorithm: we define two subdomains, $\Omega_{1}=\left(-L_{1}, 0\right)$ and $\Omega_{2}=\left(0, L_{2}\right)$, and want to couple the advection-diffusion equation in $\Omega_{1}$ with an advection equation in $\Omega_{2}$, defined by the transport operator $\mathcal{L}_{a} \equiv \partial_{t}+a \partial_{x}+c$. Our goal is to obtain a coupled solution which is as close as possible to the fully viscous solution of the original problem.

We start with the case $a>0$, where according to (2.2) the exterior boundary condition is $\mathcal{L}_{a} u\left(L_{2}, \cdot\right)=g_{2}$. Suppose there exists a decomposition $\mathcal{L}_{a d}=\widetilde{\mathcal{L}}_{m a} \mathcal{L}_{a}$ with $\mathcal{L}_{a}$ a transport operator propagating to the right, and $\widetilde{\mathcal{L}}_{m a}$ a transport operator propagating to the left. The original problem

$$
\begin{cases}\widetilde{\mathcal{L}}_{m a} \mathcal{L}_{a} u=f & \text { in } \Omega \times(0, T), \\ u\left(-L_{1}, \cdot\right)=g_{1} & \text { on }(0, T) \\ \mathcal{L}_{a} u\left(L_{2}, \cdot\right)=g_{2} & \text { on }(0, T) \\ u(\cdot, 0)=h & \text { in } \Omega\end{cases}
$$

can then be solved by introducing $u_{m a}:=\mathcal{L}_{a} u$, and solving the two problems

$$
\left\{\begin{array} { l } 
{ \widetilde { \mathcal { L } } _ { m a } u _ { m a } = f \text { in } \Omega _ { 2 } \times ( 0 , T ) , } \\
{ u _ { m a } ( L _ { 2 } , \cdot ) = g _ { 2 } \text { on } ( 0 , T ) , } \\
{ u _ { m a } ( \cdot , 0 ) = \mathcal { L } _ { a } u ( \cdot , 0 ) \text { in } \Omega _ { 2 } , }
\end{array} \quad \left\{\begin{array}{l}
\widetilde{\mathcal{L}}_{m a} \mathcal{L}_{a} u_{a d}=f \text { in } \Omega_{1} \times(0, T), \\
u_{a d}\left(-L_{1}, \cdot\right)=g_{1} \text { on }(0, T), \\
\mathcal{L}_{a} u_{a d}(0, \cdot)=u_{m a}(0, \cdot) \text { on }(0, T), \\
u_{a d}(\cdot, 0)=h \text { in } \Omega_{1},
\end{array}\right.\right.
$$


which leads to $u_{a d}=u_{\mid \Omega_{1}}$. Unfortunately, the exact factorization $\mathcal{L}_{a d}=\widetilde{\mathcal{L}}_{m a} \mathcal{L}_{a}$ is expensive, but we can use an approximation with a remainder,

$$
\mathcal{L}_{a d}=\frac{\nu}{a^{2}}\left(\mathcal{L}_{m a} \mathcal{L}_{a}-\mathcal{R}\right) \text { with } \mathcal{R}=\left(\partial_{t}+c\right)^{2} \text { and } \mathcal{L}_{m a}=\partial_{t}-a \partial_{x}+c+\frac{a^{2}}{\nu} .
$$

The viscous solution $u$ satisfies $\mathcal{L}_{m a} \mathcal{L}_{a} u=a^{2} f / \nu+\mathcal{R} u$, and the algorithm corresponding to (2.3) is

$$
\left\{\begin{array} { l } 
{ \mathcal { L } _ { m a } u _ { m a } = \frac { a ^ { 2 } } { \nu } f + \mathcal { R } u \text { in } \Omega _ { 2 } \times ( 0 , T ) , } \\
{ u _ { m a } ( L _ { 2 } , \cdot ) = g _ { 2 } \text { on } ( 0 , T ) , } \\
{ u _ { m a } ( \cdot , 0 ) = f ( \cdot , 0 ) + \nu d _ { x } ^ { 2 } h \text { in } \Omega _ { 2 } , }
\end{array} \quad \left\{\begin{array}{l}
\mathcal{L}_{a d} u_{a d}=f \text { in } \Omega_{1} \times(0, T), \\
u_{a d}\left(-L_{1}, \cdot\right)=g_{1} \text { on }(0, T), \\
\mathcal{L}_{a} u_{a d}(0, \cdot)=u_{m a}(0, \cdot) \text { on }(0, T), \\
u_{a d}(\cdot, 0)=h \text { in } \Omega_{1} .
\end{array}\right.\right.
$$

Since $u$ is unknown to evaluate the remainder, we approximate it by solving an advection equation, and our new factorization algorithm is

$$
\begin{aligned}
& \left\{\begin{array}{l}
\mathcal{L}_{a} u_{a}^{k}=f \text { in } \Omega_{2} \times(0, T) \\
u_{a}^{k}(0, \cdot)=u_{a d}^{k-1}(0, \cdot) \text { on }(0, T) \\
u_{a}^{k}(\cdot, 0)=h \text { in } \Omega_{2}
\end{array}\right. \\
& \left\{\begin{array}{l}
\mathcal{L}_{m a} u_{m a}^{k}=\frac{a^{2}}{\nu} f+\mathcal{R} u_{a}^{k} \text { in } \Omega_{2} \times(0, T), \\
u_{m a}^{k}\left(L_{2}, \cdot\right)=g_{2} \text { on }(0, T) \\
u_{m a}^{k}(\cdot, 0)=f(\cdot, 0)+\nu d_{x}^{2} h \text { in } \Omega_{2}
\end{array}\right. \\
& \left\{\begin{array}{l}
\mathcal{L}_{a d} u_{a d}^{k}=f \text { in } \Omega_{1} \times(0, T) \\
u_{a d}^{k}\left(-L_{1}, \cdot\right)=g_{1} \text { on }(0, T) \\
\mathcal{L}_{a} u_{a d}^{k}(0, \cdot)=u_{m a}^{k}(0, \cdot) \text { on }(0, T) \\
u_{a d}^{k}(\cdot, 0)=h \text { in } \Omega_{1}
\end{array}\right.
\end{aligned}
$$

where we start with a given initial guess $u_{a d}^{0}(0, \cdot)=g_{a d}^{0}$. We will prove well posedness of this algorithm in Section 3, and give precise error estimates when $\nu$ is small, which show that the new factorization algorithm gives one and a half orders of magnitude better solutions in the viscous subregion than the best other coupling algorithms from the literature.

When $a<0$, we have the factorization with remainder in reverse order, $\mathcal{L}_{a d}=$ $\frac{\nu}{a^{2}}\left(\mathcal{L}_{a} \mathcal{L}_{m a}-\mathcal{R}\right)$, and now the operator $\mathcal{L}_{a}$ propagates to the left, and $\mathcal{L}_{m a}$ to the right. The viscous solution $u$ satisfies $\mathcal{L}_{a} \mathcal{L}_{m a} u=a^{2} f / \nu+\mathcal{R} u$, and introducing $u_{a}:=\mathcal{L}_{m a} u$, the algorithm corresponding to (2.3) is

$$
\left\{\begin{array} { l } 
{ \mathcal { L } _ { a } u _ { a } = \frac { a ^ { 2 } } { \nu } f + \mathcal { R } u \text { in } \Omega _ { 2 } \times ( 0 , T ) , } \\
{ u _ { a } ( L _ { 2 } , \cdot ) = \mathcal { L } _ { m a } u ( L _ { 2 } , \cdot ) \text { on } ( 0 , T ) , } \\
{ u _ { a } ( \cdot , 0 ) = \mathcal { L } _ { m a } u ( \cdot , 0 ) \text { in } \Omega _ { 2 } , }
\end{array} \quad \left\{\begin{array}{l}
\mathcal{L}_{a d} u_{a d}=f \text { in } \Omega_{1} \times(0, T) \\
u_{a d}\left(-L_{1}, \cdot\right)=g_{1} \text { on }(0, T) \\
\mathcal{L}_{m a} u_{a d}(0, \cdot)=u_{a}(0, \cdot) \text { on }(0, T) \\
u_{a d}(\cdot, 0)=h \text { in } \Omega_{1}
\end{array}\right.\right.
$$

Since $u$ is unknown to evaluate the remainder and the boundary conditions, we approximate it again by solving an advection equation, and our new factorization algorithm 
becomes

$$
\begin{aligned}
& \left\{\begin{array}{l}
\mathcal{L}_{a} u_{a}^{1}=f \text { in } \Omega_{2} \times(0, T), \\
u_{a}^{1}\left(L_{2}, \cdot\right)=g_{2} \text { on }(0, T), \\
u_{a}^{1}(\cdot, 0)=h \text { in } \Omega_{2}
\end{array}\right. \\
& \left\{\begin{array}{l}
\mathcal{L}_{a} u_{a}^{2}=\frac{a^{2}}{\nu} f+\mathcal{R} u_{a}^{1} \text { in } \Omega_{2} \times(0, T), \\
u_{a}^{2}\left(L_{2}, \cdot\right)=\mathcal{L}_{m a} u_{a}^{1}\left(L_{2}, \cdot\right) \text { on }(0, T), \\
u_{a}^{2}(\cdot, 0)=\mathcal{L}_{m a} u_{a}^{1}(\cdot, 0) \text { in } \Omega_{2}
\end{array}\right. \\
& \left\{\begin{array}{l}
\mathcal{L}_{a d} u_{a d}=f \text { in } \Omega_{1} \times(0, T) \\
u_{a d}\left(-L_{1}, \cdot\right)=g_{1} \text { on }(0, T) \\
\mathcal{L}_{m a} u_{a d}(0, \cdot)=u_{a}^{2}(0, \cdot) \text { on }(0, T), \\
u_{a d}(\cdot, 0)=h \text { in } \Omega_{1}
\end{array}\right.
\end{aligned}
$$

where one could also directly compute $\mathcal{L}_{m a} u_{a}^{1}\left(L_{2}, \cdot\right)=2 g_{2}^{\prime}+\left(2 c+a^{2} / \nu\right) g_{2}-f\left(L_{2}, \cdot\right)$ and $\mathcal{L}_{m a} u_{a}^{1}(\cdot, 0)=f(\cdot, 0)-2 a d_{x} h+a^{2} h / \nu$. There is no iteration for $a<0$ in the algorithm, because the boundary condition $g_{2}$ at $x=L_{2}$ in the first step can not be updated naturally from the viscous solution $u_{a d}$ in $\Omega_{1}$. We will study this algorithm in detail in Section 4 and show that it gives an order of magnitude better solutions in the viscous subregion than the other coupling algorithms from the literature.

2.3. Well-posedness results for advection reaction diffusion problems. We work in the usual Sobolev spaces in time and space, $H^{s}(0, T)$ and $H^{s}(\Omega)$ for $\Omega \subset \mathbb{R}$, $H^{s}(\Omega \times(0, T))$ in the hyperbolic case, and the anisotropic spaces $H^{r, s}(\Omega \times(0, T))$ in the parabolic case. For clarity, we will add an index defining time or space in the Sobolev space, for instance $H_{t}^{s} \equiv H^{s}(0, T)$. We introduce for any domain $\Omega \subset \mathbb{R}$ the anisotropic Sobolev spaces (see [28])

$$
H^{r, s}(\Omega \times(0, T))=L^{2}\left(0, T ; H^{r}(\Omega)\right) \cap H^{s}\left(0, T ; L^{2}(\Omega)\right) .
$$

If $u$ is in $H^{r, s}(\Omega \times(0, T))$, then for any integer $j$ and $k$, we have

$$
\frac{\partial^{j}}{\partial x^{j}} \frac{\partial^{k}}{\partial t^{k}} u \in H^{\mu, \nu}(\Omega \times(0, T)), \quad \text { where } \frac{\mu}{r}=\frac{\nu}{s}=1-\left(\frac{j}{r}+\frac{k}{s}\right) .
$$

We introduce the space $V^{r, s}$ of traces of functions in $H^{r, s}(\Omega \times(0, T))$ for the half-space $\Omega=\mathbb{R}^{-}$(and similarly for $\Omega=\mathbb{R}^{+}$). Denoting by $f_{k}$ the trace of the k-th derivative in time on the initial line, $x \in \mathbb{R}^{-}$, and by $g_{j}$ the trace of the $\mathrm{j}$-th derivative in space on the boundary $x=0, t \in(0, T)$, the trace space $V^{r, s}$ is defined by

$$
\begin{gathered}
V^{r, s}:=\left\{\left(f_{k}, g_{j}\right) \in \prod_{k<s-\frac{1}{2}} H^{p_{k}}(\Omega) \times \prod_{j<r-\frac{1}{2}} H^{\mu_{j}}(0, T),\right. \\
p_{k}=\frac{r}{s}\left(s-k-\frac{1}{2}\right), \quad \mu_{j}=\frac{s}{r}\left(r-j-\frac{1}{2}\right), \\
\frac{\partial^{k} g_{j}}{\partial t^{k}}(0)=\frac{\partial^{j} f_{k}}{\partial x^{j}}(0), \quad \text { if } \frac{j}{r}+\frac{k}{s}<1-\frac{1}{2}\left(\frac{1}{r}+\frac{1}{s}\right), \\
\left.\int_{0}^{\infty}\left|\frac{\partial^{j} f_{k}}{\partial x^{j}}\left(\sigma^{s}\right)-\frac{\partial^{k} g_{j}}{\partial t^{k}}\left(\sigma^{r}\right)\right|^{2} \frac{d \sigma}{\sigma}<\infty, \quad \text { if } \frac{j}{r}+\frac{k}{s}=1-\frac{1}{2}\left(\frac{1}{r}+\frac{1}{s}\right)\right\} .
\end{gathered}
$$

TheOREM $2.1([28])$. For positive real numbers $r$ and $s$ such that $1-\frac{1}{2}\left(\frac{1}{r}+\frac{1}{s}\right)>0$, the trace map

$$
u \mapsto\left\{\left\{\frac{\partial^{k} u}{\partial t^{k}}(x, 0)\right\}_{k<s-\frac{1}{2}},\left\{\frac{\partial^{j} u}{\partial x^{j}}(0, t)\right\}_{j<r-\frac{1}{2}}\right\}
$$


is defined and continuous from $H^{r, s}(\Omega \times(0, T))$ onto $V^{r, s}$.

We start with well-posedness results for the advection equation, by stating a general result, applicable to $\mathcal{L}_{a}$ in $\Omega$ or $\Omega_{2}$, and $\mathcal{L}_{m a}$ in $\Omega_{2}$. To this end, we introduce $\mathcal{O}=\left(x_{1}, x_{2}\right)$ and consider

$$
\mathcal{L}_{b} v:=\partial_{t} v+b \partial_{x} v+\eta v=p \text { in } \mathcal{O} \times(0, T) .
$$

Let $\mathcal{M}_{b}$ be the spatial part of the operator $\mathcal{L}_{b}$, i.e. $\mathcal{L}_{b}=\partial_{t}+\mathcal{M}_{b}$. We denote the boundary point where the flux enters the domain by $x^{-}$, the other boundary point by $x^{+}$, and define the characteristic time $\tau(x):=\inf \{t \geq 0$, s.t. $x-a t \notin \overline{\mathcal{O}}\}$. If $b>0$, $x^{-}=x_{1}$ and $\tau(x)=\frac{x-x_{1}}{b}$, and if $b<0, x^{-}=x_{2}$ and $\tau(x)=\frac{x-x_{2}}{b}$. Note that $\tau$ is a continuous function of $x$. We therefore equip (2.11) with initial and boundary conditions

$$
v(\cdot, 0)=h, \quad v\left(x^{-}, \cdot\right)=g .
$$

The following well-posedness result can be found in 31.

Theorem 2.2. If $p \in L^{2}(\mathcal{O} \times(0, T)), g \in L^{2}(0, T)$ and $v_{0} \in L^{2}(\mathcal{O})$, then the transport problem (2.112.12) has a unique weak solution $v \in L_{x, t}^{2}$, given by (the characteristic function of $\omega$ in $\mathbb{R}^{2}$ is denoted by $\mathbf{1}_{\omega}$ )

$$
\begin{gathered}
v(x, t)=h(x-b t) e^{-\eta t} \mathbf{1}_{t<\tau(x)}+g(t-\tau(x)) e^{-\eta \tau(x)} \mathbf{1}_{t>\tau(x)} \\
+\int_{(t-\tau(x))^{+}}^{t} p(x-b(t-s), s) e^{-\eta(t-s)} d s .
\end{gathered}
$$

If for some $\gamma>0$ we have $h \in H^{\gamma}(\mathcal{O}), g \in H^{\gamma}(0, T)$ and $p \in H^{\gamma}(\mathcal{O} \times(0, T))$, with the compatibility conditions

$$
d_{t}^{k} g(0)=\left(\sum_{j=0}^{k-1}\left(-\mathcal{M}_{b}\right)^{j} \partial_{t}^{k-1-j} p\right)\left(x^{-}, 0\right)+\left(-\mathcal{M}_{b}\right)^{k} h\left(x^{-}\right) \quad \text { for } \quad 0 \leq k \leq \gamma-1,
$$

then $v \in H^{\gamma}(\mathcal{O} \times(0, T))$ and $v\left(x^{+}, \cdot\right) \in H^{\gamma}(0, T)$. Furthermore, we have for $0 \leq k \leq \gamma$ the estimates

$$
\eta\left\|\partial_{t}^{k} v\right\|_{L_{x, t}^{2}}^{2}+|b|\left\|\partial_{t}^{k} v\left(x^{+}, \cdot\right)\right\|_{L_{t}^{2}}^{2} \leq \frac{1}{\eta}\left\|\partial_{t}^{k} p\right\|_{L_{x, t}^{2}}^{2}+\left\|\partial_{t}^{k} v(\cdot, 0)\right\|_{L_{x}^{2}}^{2}+|b|\left\|d_{t}^{k} g\right\|_{L_{t}^{2}}^{2} .
$$

Similarly, we also use well-posedness results for the advection reaction diffusion equation

$$
\begin{aligned}
\mathcal{L}_{a d} u:=\partial_{t} u-\nu \partial_{x}^{2} u+a \partial_{x} u+c u & =f & & \text { in } \mathcal{O} \times(0, T), \\
\mathcal{B}_{1} u\left(x_{1}, \cdot\right) & =g_{1} & & \text { on }(0, T), \\
\mathcal{B}_{2} u\left(x_{2}, \cdot\right) & =g_{2} & & \text { on }(0, T), \\
u(x, 0) & =h & & \text { in } \mathcal{O},
\end{aligned}
$$

with boundary operators according to (2.2). We define $\mathcal{M}_{a d}$ to be the spatial part of the operator $\mathcal{L}_{a d}$, i.e. $\mathcal{L}_{a d}=\partial_{t}+\mathcal{M}_{a d}$.

TheOrem 2.3. For $\gamma>0$, let $f \in H^{2 \gamma, \gamma}(\mathcal{O} \times(0, T)), g_{1} \in H_{t}^{\gamma+\frac{3}{4}}, g_{2} \in H_{t}^{\gamma+\frac{3}{4}}$ for negative $a$, and $g_{2} \in H_{t}^{\gamma-\frac{1}{4}}$ for positive $a, h \in H_{x}^{2 \gamma+1}(\mathcal{O})$, with the compatibility conditions for $0 \leq k<\gamma-\frac{1}{2}$ and $0 \leq k^{\prime}<\gamma-\frac{3}{2}$ for negative a given by

$$
1 \leq j \leq 2, \quad d_{t}^{k} g_{j}(0)=\left(-\mathcal{M}_{a d}\right)^{k} h\left(x_{j}\right)+\left(\sum_{j=0}^{k-1}\left(-\mathcal{M}_{a d}\right)^{j} \partial_{t}^{k-1-j} f\right)\left(x_{j}, 0\right),
$$


and for positive a the second compatibility condition is replaced by

$$
d_{t}^{k^{\prime}} g_{2}(0)-\nu \sum_{j=0}^{k^{\prime}-1}\left(-\mathcal{M}_{a d}\right)^{j} \partial_{t}^{k^{\prime}-1-j} \partial_{x}^{2} f\left(x_{2}, 0\right)-\nu d_{x}^{2}\left(-\mathcal{M}_{a d}\right)^{k^{\prime}} h\left(x_{2}\right)=\partial_{t}^{k^{\prime}} f\left(x_{2}, 0\right),
$$

then problem (2.16) has a unique solution $u$ in $H^{2(\gamma+1), \gamma+1}(\mathcal{O} \times(0, T))$.

Proof. Existence and regularity results are well-known for Dirichlet boundary conditions on both sides, see 28, 31, so we do not consider the case of negative advection further. In 31 more precise results with error bounds in $\nu$ for the hyperbolic equation (see Theorem 3.3) can be found. In the case where $a>0$, due to the absorbing boundary, we need to modify the proof on the right boundary, and we use a Fourier transform in time. A weak solution is obtained by a variational formulation, like in [30, 4] for instance. The regularity is obtained as follows: we first modify the boundary condition in (2.16) on the right at $x=x_{2}$ to Dirichlet data $\tilde{g}_{2} \in H_{t}^{\gamma+\frac{3}{4}}$. Because of the compatibility conditions on the left, and imposing symmetric compatibility conditions on $\tilde{g}_{2}$ on the right, there is a unique solution $\tilde{u} \in H^{2(\gamma+1),(\gamma+1)}(\mathcal{O} \times(0, T))$, see [28. The difference $v=u-\tilde{u}$ is solution of the homogeneous case of equation (3.1), but the boundary condition on the right becomes $\mathcal{L}_{a}(u-\tilde{u})=q_{2}:=g_{2}-\mathcal{L}_{a} \tilde{u}$. By the regularity results above, $q_{2}$ is in $H_{t}^{\gamma-\frac{1}{4}}$. To estimate $v$, we will make use of the Fourier transform. We extend all functions by 0 in $\mathbb{R}_{-}$, and smoothly into $(T,+\infty)$, and define

$$
\hat{v}(\omega)=\frac{1}{2 \pi} \int_{\mathbb{R}} e^{-i \omega t} v(t) d t .
$$

Since the initial value vanishes, the equation is Fourier transformed in time to

$$
\widehat{\mathcal{L}}_{a d} \hat{v}:=-\nu \partial_{x}^{2} \hat{v}+a \partial_{x} \hat{v}+(c+i \omega) \hat{v}=0 \text { on } \mathcal{O} \times \mathbb{C} .
$$

This is for each $\omega$ an ordinary differential equation, with characteristic roots

$$
\lambda_{+}(\omega)=\frac{1}{2 \nu}\left(a+\sqrt{a^{2}+4 \nu(c+i \omega)}\right), \quad \lambda_{-}(\omega)=\frac{1}{2 \nu}\left(a-\sqrt{a^{2}+4 \nu(c+i \omega)}\right),
$$

with $\operatorname{Re}\left(\lambda_{+}\right)>0$ and $\operatorname{Re}\left(\lambda_{-}\right)<0$. The general solution is

$$
\hat{v}(x, \omega)=\ell_{+}(\omega) e^{\lambda_{+} x}+\ell_{-}(\omega) e^{\lambda_{-} x} .
$$

Using the boundary conditions, we then get the solution

$$
\hat{v}(x, \omega)=\hat{q}_{2}(\omega) \frac{e^{\lambda_{+}\left(x-x_{1}\right)}-e^{\lambda_{-}\left(x-x_{1}\right)}}{\nu \lambda_{+}^{2} e^{\lambda_{+}\left(x_{2}-x_{1}\right)}-\nu \lambda_{-}^{2} e^{\lambda_{-}\left(x_{2}-x_{1}\right)}},
$$

where we have used the relation $c+i \omega+a \lambda_{ \pm}=\nu\left(\lambda_{ \pm}\right)^{2}$. The value at $x=x_{2}$ can be equivalently written as

$$
\hat{v}\left(x_{2}, \omega\right)=\hat{q}_{2}(\omega) \frac{e^{-\left(\lambda_{+}-\lambda_{-}\right)\left(x_{2}-x_{1}\right)}-1}{\nu \lambda_{+}^{2}\left(\left(\frac{\lambda_{-}}{\lambda_{+}}\right)^{2} e^{-\left(\lambda_{+}-\lambda_{-}\right)\left(x_{2}-x_{1}\right)}-1\right)} .
$$

In order to estimate the regularity of $v\left(x_{2}, \cdot\right)$, we need to estimate the multiplicative factor on the right for large $\omega$. We can see from (2.19) that $\lambda_{+}(\omega) \sim-\lambda_{-}(\omega) \sim \sqrt{\frac{i \omega}{\nu}}$. 
Therefore $\left|\hat{v}\left(x_{2}, \omega\right)\right| \sim\left|\frac{\hat{q}_{2}(\omega)}{\nu \lambda_{+}^{2}}\right| \sim\left|\frac{\hat{q}_{2}(\omega)}{\omega}\right|$. Since $q_{2} \in H_{t}^{\gamma-\frac{1}{4}}$, we conclude that $v\left(x_{2}, \cdot\right) \in$ $H_{t}^{\gamma+\frac{3}{4}}$. Then $v$ is solution of the advection-diffusion equation with Dirichlet boundary conditions, and the data has sufficient regularity to conclude.

3. Properties of the factorization algorithm for positive advection. We consider the advection-diffusion equation in $\Omega=\left(-L_{1}, L_{2}\right)$ with Dirichlet boundary condition on the left, and absorbing boundary condition given by the transport operator on the right (see [26]),

$$
\begin{aligned}
\mathcal{L}_{a d} u:=\partial_{t} u-\nu \partial_{x}^{2} u+a \partial_{x} u+c u & =f & & \text { in } \Omega \times(0, T), \\
u\left(-L_{1}, \cdot\right) & =g_{1} & & \text { on }(0, T), \\
\mathcal{L}_{a} u\left(L_{2}, \cdot\right) & =g_{2} & & \text { on }(0, T), \\
u(\cdot, 0) & =h & & \text { in } \Omega .
\end{aligned}
$$

We suppose in this section that $f$ and $g_{1}$ are compactly supported in $(0, T]$, that $h$ is compactly supported in $\Omega_{1}=\left(-L_{1}, 0\right)$, and that for each $t$ the function $f(\cdot, t)$ is compactly supported in $\Omega$. We further assume that the boundary condition at $L_{2}$ is absorbing, that is $g_{2}=0$. Therefore the compatibility conditions are satisfied to any order on both ends of the interval $\Omega$, and for $f \in H^{\frac{9}{2}, \frac{9}{4}}(\Omega \times(0, T)), h \in H_{x}^{\frac{11}{2}}$, and $g_{1} \in H_{t}^{3}, u$ is defined in $H^{\frac{13}{2}, \frac{13}{4}}(\Omega \times(0, T))$.

3.1. Well-posedness. The remainder $\mathcal{R}$ for computing $u_{m a}^{k}$ in the new factorization algorithm (2.5) contains two time derivatives, which lead to an important loss of regularity at each iteration. We will however see that the error order in $\nu$ can not be improved further after two iterations, and hence we only study the first two iterations in detail. We start with the well-posedness of the algorithm.

Algorithm (2.5) starts with an initial guess $g_{a d}^{0}$ as boundary condition for $u_{a}$. We assume that $g_{a d}^{0} \in H_{t}^{4}$ and is compactly supported in $(0, T]$. Using that $f \in$ $H^{4}\left(\Omega_{2} \times(0, T)\right)$, that $h$ vanishes in $\Omega_{2}$, and that the compatibility conditions at $x=0, t=0$ are satisfied, the solution of

$$
\mathcal{L}_{a} u_{a}^{1}=f \text { in } \Omega_{2}, \quad u_{a}^{1}(0, \cdot)=g_{a d}^{0}, \quad u_{a}^{1}(\cdot, 0)=0
$$

satisfies $u_{a}^{1} \in H^{4}\left(\Omega_{2} \times(0, T)\right)$.

The right hand side for the modified advection equation in (2.5) is then $f_{m a}^{1}=$ $\frac{a^{2}}{\nu} f+\mathcal{R} u_{a}^{1} \in H^{2}\left(\Omega_{2} \times(0, T)\right)$, and solving

$$
\mathcal{L}_{m a} u_{m a}^{1}=f_{m a}^{1} \text { in } \Omega_{2}, \quad u_{m a}^{1}\left(L_{2}, \cdot\right)=0, \quad u_{m a}^{1}(\cdot, 0)=0
$$

the compatibility conditions at $x=L_{2}$ are again satisfied to any order, which implies that $u_{m a}^{1} \in H^{2}\left(\Omega_{2} \times(0, T)\right)$ and $u_{m a}^{1}(0, \cdot) \in H^{2}(0, T)$. The latter then becomes the right boundary data for the advection diffusion problem in $\Omega_{1}$,

$$
\mathcal{L}_{a d} u_{a d}^{1}=f \text { in } \Omega_{1}, \quad u_{a d}^{1}\left(-L_{1}, \cdot\right)=g_{1}, \quad \mathcal{L}_{a} u_{a d}^{1}(0, \cdot)=u_{m a}^{1}(0, \cdot), \quad u_{a d}^{1}(\cdot, 0)=h .
$$

We have seen already that the compatibility conditions on the left are satisfied, and on the right, at the corner $(0,0)$, with the regularity of $u_{m a}^{1}$, the condition $u_{m a}^{1}(0,0)-$ $\nu d_{x}^{2} h(0)=f(0,0)$ holds, since both sides of this equality vanish. Since $f \in H^{\frac{9}{2}}, \frac{9}{4}(\Omega \times$ $(0, T)), h \in H_{x}^{\frac{11}{2}}, g_{1} \in H_{t}^{3}$, and $u_{m a}^{1}(0, \cdot) \in H_{t}^{2}$, we obtain $u_{a d}^{1} \in H^{\frac{13}{2}}, \frac{13}{4}\left(\Omega_{1} \times(0, T)\right)$ and $u_{a d}^{1}(0, \cdot) \in H_{t}^{3}$, and at the corner $(0,0)$, we have for $g_{a d}^{1}:=u_{a d}^{1}(0, \cdot)$

$$
\begin{aligned}
g_{a d}^{1}(0)=h(0), \quad d_{t} g_{a d}^{1}(0)+\mathcal{M}_{a d} h(0) & =f(0,0), \\
d_{t}^{2} g_{a d}^{1}(0)-\mathcal{M}_{a d}^{2} h(0) & =\partial_{t} f(0,0)-\mathcal{M}_{a d} f(0,0) .
\end{aligned}
$$


We now start the second iteration with the computation of $u_{a}^{2}$, using $u_{a}^{1}(0, \cdot)=g_{a d}^{1}=$ $u_{a d}^{1}(0, \cdot) \in H_{t}^{3}$. Since $h$ is compactly supported in $\Omega_{1}, \mathcal{M}_{a d}^{p} h(0)=\mathcal{M}_{a}^{p} h(0)=0$, and (3.2) are appropriate compatibility conditions to compute $u_{a}^{2} \in H^{3}\left(\Omega_{2} \times(0, T)\right)$. We define the new right hand side

$$
f_{m a}^{2}=\frac{a^{2}}{\nu} f+\mathcal{R} u_{a}^{2} \in H^{1}\left(\Omega_{2} \times(0, T)\right),
$$

and compute the solution of

$$
\mathcal{L}_{m a} u_{m a}^{2}=f_{m a}^{2} \text { in } \Omega_{2}, \quad u_{m a}^{2}\left(L_{2}, \cdot\right)=0, \quad u_{m a}^{2}(\cdot, 0)=0 .
$$

We thus obtain $u_{m a}^{2} \in H^{1}\left(\Omega_{2} \times(0, T)\right)$ and $u_{m a}^{2}(0, \cdot) \in H^{1}(0, T)$. The last step of the second iteration is to compute $u_{a d}^{2}$ solution of

$$
\mathcal{L}_{a d} u_{a d}^{2}=f \text { in } \Omega_{1}, \quad u_{a d}^{1}\left(-L_{1}, \cdot\right)=g_{1}, \quad \mathcal{L}_{a} u_{a d}^{1}(0, \cdot)=u_{m a}^{2}(0, \cdot), \quad u_{a d}^{1}(\cdot, 0)=h,
$$

and we need only to satisfy a compatibility condition on the left, which implies that $u_{\text {ad }}^{2} \in H^{\frac{9}{2}, \frac{9}{4}}(\Omega \times(0, T))$.

3.2. Error estimates for the factorization algorithm. We present now asymptotic error estimates for small viscosity $\nu$ when $u$, the viscous solution of (3.1), is approximated by $\left(u_{a d}^{k}, u_{a}^{k}\right)$, the solution obtained by our new factorization algorithm (2.5). We define the error quantities $e_{a}^{k}:=u_{a}^{k}-u, e_{a d}^{k}:=u_{a d}^{k}-u$, $e_{m a}^{k}:=u_{m a}^{k}-\mathcal{L}_{a} u:=u_{m a}^{k}-u_{m a}$, and suppose that all our data is $\mathcal{C}^{\infty}$ in all variables. The error equations are

$$
\left\{\begin{array} { l } 
{ \mathcal { L } _ { a } e _ { a } ^ { k } = - \nu \partial _ { x } ^ { 2 } u \text { in } \Omega _ { 2 } , } \\
{ e _ { a } ^ { k } ( 0 , \cdot ) = e _ { a d } ^ { k - 1 } ( 0 , \cdot ) , } \\
{ e _ { a } ^ { k } ( \cdot , 0 ) = 0 , }
\end{array} \quad \left\{\begin{array} { l } 
{ \mathcal { L } _ { m a } e _ { m a } ^ { k } = \mathcal { R } e _ { a } ^ { k } \text { in } \Omega _ { 2 } , } \\
{ e _ { m a } ^ { k } ( L _ { 2 } , \cdot ) = 0 , } \\
{ e _ { m a } ^ { k } ( \cdot , 0 ) = 0 , }
\end{array} \quad \left\{\begin{array}{l}
\mathcal{L}_{a d} e_{a d}^{k}=0 \text { in } \Omega_{1}, \\
e_{a d}^{k}\left(-L_{1}, \cdot\right)=0, \\
\mathcal{L}_{a} e_{a d}^{k}(0, \cdot)=e_{m a}^{k}(0, \cdot), \\
e_{a d}^{k}(\cdot, 0)=0,
\end{array}\right.\right.\right.
$$

with $e_{a d}^{0}(0, \cdot):=g_{a d}^{0}-u(0, \cdot)$. We need more precise estimates than those provided by Theorems 2.2 and 2.3 First, we state precisely the initial conditions for all the equations involved: the parabolic problems in $\Omega$ and $\Omega_{1}$ will use

$$
\partial_{t}^{k} u(\cdot, 0)=\left(-\mathcal{M}_{a d}\right)^{k} h \quad \text { and } \quad \partial_{t}^{k} u_{a d}^{p}(\cdot, 0)=\left(-\mathcal{M}_{a d}\right)^{k} h,
$$

the forward hyperbolic problem in $\Omega_{2}$ uses

$$
\partial_{t}^{k} u_{a}^{p}(\cdot, 0)=\left(-\mathcal{M}_{a}\right)^{k} h=0,
$$

and the backward hyperbolic problem in $\Omega_{2}$ uses

$$
\partial_{t}^{k} u_{m a}^{p}(\cdot, 0)=\sum_{j=0}^{k-1}\left(-\mathcal{M}_{m a}\right)^{j} \partial_{t}^{k-1-j} f_{m a}^{p}(\cdot, 0)+\left(-\mathcal{M}_{m a}\right)^{k} u_{m a, 0}=0 .
$$

The solution of the exact backward hyperbolic problem in $\Omega_{2}$ has as initial condition

$$
\partial_{t}^{k} u_{m a}(\cdot, 0)=\sum_{j=0}^{k-1}\left(-\mathcal{M}_{m a}\right)^{j} \partial_{t}^{k-1-j} f_{m a}(\cdot, 0)+\left(-\mathcal{M}_{m a}\right)^{k} u_{m a, 0}=0,
$$


from which we infer the initial values for the errors such that

$$
\partial_{t}^{k} e_{a d}^{p}(\cdot, 0)=0 \text { in } \Omega_{1}, \quad \partial_{t}^{k} e_{a}^{p}(\cdot, 0)=0 \text { and } \partial_{t}^{k} e_{m a}^{p}(\cdot, 0)=0 \text { in } \Omega_{2} .
$$

We start with estimates for the solution of the advection-diffusion equation (3.1) with vanishing initial data and vanishing boundary data $g_{1}$. A first lemma gives results for the problem with $g_{2}=0$, based on energy estimates, and a second lemma gives estimates where only the right-hand side $f$ is non-zero.

Lemma 3.1. Suppose that $a>0$, and that $h$ vanishes identically in $\Omega, g_{1}$ and $g_{2}$ vanish on $(0, T)$, and that $f$ is in $\mathcal{C}_{0}^{\infty}(\Omega \times(0, T])$. Then there is a positive constant $C$ such that for any $\nu>0$, and any $k \leq \gamma$, the solution $u_{1}$ of (3.1) satisfies the estimates

$$
\begin{aligned}
\left\|\partial_{t}^{k} u_{1}\right\|_{L_{x, t}^{2}}^{2}+\left\|\partial_{t}^{k} u_{1}\left(L_{2}, \cdot\right)\right\|_{L_{t}^{2}}^{2}+\nu\left\|\partial_{t}^{k} \partial_{x} u_{1}\right\|_{L_{x, t}^{2}}^{2} \leq C\left\|\partial_{t}^{k} f\right\|_{L_{x, t}^{2}}^{2} \\
\nu\left\|\partial_{t}^{k} \partial_{x}^{2} u_{1}\right\|_{L_{x, t}^{2}} \leq\left\|\partial_{t}^{k} f\right\|_{L_{x, t}^{2}}
\end{aligned}
$$

Proof. Since the compatibility conditions are satisfied, $u_{1}$ is in $H^{\infty}$, and the initial value of $\partial_{t}^{k} u_{1}$ vanishes as well. We start with $k=0$ : multiplying the equation by $u_{1}$ and integrating over $\Omega$, taking into account that $u_{1}$ vanishes at $-L_{1}$ gives

$$
\begin{aligned}
\frac{1}{2} \frac{d}{d t}\left\|u_{1}(\cdot, t)\right\|_{L_{x}^{2}}^{2} & +c\left\|u_{1}(\cdot, t)\right\|_{L_{x}^{2}}^{2}+\nu\left\|\partial_{x} u_{1}(\cdot, t)\right\|_{L_{x}^{2}}^{2} \\
& +\frac{a}{2} u_{1}^{2}\left(L_{2}, t\right)-\nu\left(u_{1} \partial_{x} u_{1}\right)\left(L_{2}, t\right)=\int_{\Omega} f(x, t) u_{1}(x, t) d x .
\end{aligned}
$$

Using the boundary condition at $L_{2}$ yields

$$
\begin{aligned}
\frac{1}{2} \frac{d}{d t}\left(\left\|u_{1}(\cdot, t)\right\|_{L_{x}^{2}}^{2}\right. & \left.+\frac{\nu}{a} u_{1}^{2}\left(L_{2}, t\right)\right)+c\left\|u_{1}(\cdot, t)\right\|_{L_{x}^{2}}^{2}+\nu\left\|\partial_{x} u_{1}(\cdot, t)\right\|_{L_{x}^{2}}^{2} \\
& +\left(\frac{a}{2}+\frac{\nu c}{a}\right) u_{1}^{2}\left(L_{2}, t\right)=\int_{\Omega} f(x, t) u_{1}(x, t) d x,
\end{aligned}
$$

and by Cauchy-Schwarz and Young's inequality we obtain

$$
\int_{\Omega} f(x, t) u_{1}(x, t) d x \leq\left\|u_{1}(\cdot, t)\right\|_{L_{x}^{2}}\|f(\cdot, t)\|_{L_{x}^{2}} \leq \frac{c}{2}\left\|u_{1}(\cdot, t)\right\|_{L_{x}^{2}}^{2}+\frac{1}{2 c}\|f(\cdot, t)\|_{L_{x}^{2}}^{2} .
$$

Integrating in time over $(0, T)$, and dropping the first term which is positive, we obtain, since the initial data vanishes, the inequality

$$
c\left\|u_{1}\right\|_{L_{x, t}^{2}}^{2}+2 \nu\left\|\partial_{x} u_{1}\right\|_{L_{x, t}^{2}}^{2}+a\left\|u_{1}\left(L_{2}, \cdot\right)\right\|_{L_{t}^{2}}^{2} \leq \frac{1}{c}\|f\|_{L_{x, t}^{2}}^{2},
$$

which proves (3.5) for $k=0$. To prove (3.6), we multiply the equation by $-\partial_{x}^{2} u_{1}$ and integrate in $x$,

$$
\begin{aligned}
\nu\left\|\partial_{x}^{2} u_{1}(\cdot, t)\right\|_{L_{x}^{2}}^{2}-\left(\partial_{t} u_{1}(\cdot, t), \partial_{x}^{2} u_{1}(\cdot, t)\right) & -a\left(\partial_{x} u_{1}(\cdot, t), \partial_{x}^{2} u_{1}(\cdot, t)\right) \\
& -c\left(u_{1}(\cdot, t), \partial_{x}^{2} u_{1}(\cdot, t)\right)=-\left(f(\cdot, t), \partial_{x}^{2} u_{1}(\cdot, t)\right) .
\end{aligned}
$$

An integration by parts leads to

$$
\begin{aligned}
\nu\left\|\partial_{x}^{2} u_{1}(\cdot, t)\right\|_{L_{x}^{2}}^{2}+\frac{1}{2} \frac{d}{d t}\left\|\partial_{x} u_{1}(\cdot, t)\right\|_{L_{x}^{2}}^{2} & +c\left\|\partial_{x} u_{1}(\cdot, t)\right\|_{L_{x}^{2}}^{2}-\left[\partial _ { x } u _ { 1 } ( \cdot , t ) \left(\partial_{t} u_{1}+\frac{a}{2} \partial_{x} u_{1}\right.\right. \\
& \left.\left.+c u_{1}\right)(\cdot, t)\right]_{-L_{1}}^{L_{2}}=-\left(f(\cdot, t), \partial_{x}^{2} u_{1}(\cdot, t)\right) .
\end{aligned}
$$


By the boundary conditions, the boundary terms become

$$
a\left(\partial_{x} u_{1}\right)^{2}\left(-L_{1}, t\right)+\frac{1}{a}\left(\partial_{t} u_{1}+c u_{1}\right)^{2}\left(L_{2}, t\right)>0 .
$$

We can now integrate in time and use Cauchy-Schwarz and Young's inequality to obtain

$$
\nu\left\|\partial_{x}^{2} u_{1}\right\|_{L_{x, t}^{2}}^{2} \leq \frac{1}{\nu}\|f\|_{L_{x, t}^{2}}^{2} .
$$

The estimates with the time derivative are obtained by applying the equation to $\partial_{t}^{k} u$. (

Lemma 3.2. Assume that $a>0$. Then there are constants $\bar{\nu}>0$ and $C>0$ such that for $\nu \leq \bar{\nu}$, and for any $g_{2} \in \mathcal{C}_{0}^{\infty}((0, T])$, the solution $u_{2}$ of (3.1) with zero data $h, f$ and $g_{1}$ satisfies for all $k \leq \gamma$ the inequalities

$$
\begin{array}{r}
\forall x \in\left[-L_{1}, L_{2}\right],\left\|\partial_{t}^{k} u_{2}(x, \cdot)\right\|_{L_{t}^{2}} \leq C \nu\left\|g_{2}\right\|_{H_{t}^{k}}, \\
\left\|\partial_{t}^{k} u_{2}\right\|_{L_{x, t}^{2}} \leq C \nu^{\frac{3}{2}}\left\|g_{2}\right\|_{H_{t}^{k}}, \quad\left\|\partial_{x} \partial_{t}^{k} u_{2}\right\|_{L_{x, t}^{2}} \leq C \nu^{\frac{1}{2}}\left\|g_{2}\right\|_{H_{t}^{k}}, \\
\left\|\partial_{x}^{2} \partial_{t}^{k} u_{2}\right\|_{L_{x, t}^{2}} \leq C \nu^{-\frac{1}{2}}\left\|g_{2}\right\|_{H_{t}^{k}} .
\end{array}
$$

Proof. We use a Fourier transform argument as in the proof of Theorem 2.3. and rewrite (2.20) as

$$
\hat{u}_{2}(x, \omega)=\hat{g}_{2}(\omega) e^{\lambda_{+}\left(x-L_{2}\right)} \frac{e^{-\left(\lambda_{+}-\lambda_{-}\right)\left(x+L_{1}\right)}-1}{\nu \lambda_{+}^{2}\left(\left(\frac{\lambda_{-}}{\lambda_{+}}\right)^{2} e^{-\left(\lambda_{+}-\lambda_{-}\right)\left(L_{2}+L_{1}\right)}-1\right)} .
$$

Now we search for estimates in $\nu$ that are uniform in $\omega$. We have for the roots $\lambda_{ \pm}$the estimates

$$
\left|\lambda_{-} / \lambda_{+}\right|<1, \quad \operatorname{Re}\left(\lambda_{+}-\lambda_{-}\right) \geq a / \nu, \quad\left|\lambda_{+}\right| \geq a / \nu .
$$

The numerator in (3.8) is bounded by 2. A lower bound for the denominator is obtained by writing $\left|\nu \lambda_{+}^{2}\right| \geq \frac{a^{2}}{\nu}$ together with

$$
\left|1-\left(\frac{\lambda_{-}}{\lambda_{+}}\right)^{2} e^{-\left(\lambda_{+}-\lambda_{-}\right)\left(L_{2}+L_{1}\right)}\right| \geq 1-\left|\frac{\lambda_{-}}{\lambda_{+}}\right|^{2} e^{-R e\left(\lambda_{+}-\lambda_{-}\right)\left(L_{2}+L_{1}\right)} \geq 1-e^{-\frac{a}{\nu}\left(L_{2}+L_{1}\right)} .
$$

Inserting these estimates into (3.8) gives

$$
\left|\hat{u}_{2}(x, \omega)\right| \leq \frac{2 \nu}{a^{2}}\left|\hat{g}_{2}(\omega)\right| e^{R e \lambda_{+}\left(x-L_{2}\right)} \frac{1}{1-e^{-\frac{a}{\nu}\left(L_{2}+L_{1}\right)}} .
$$

Since $1 /|1-\mu|<2$ for $\mu<1 / 2$, for $\nu$ sufficiently small so that $e^{-\frac{a}{\nu}\left(L_{2}+L_{1}\right)}<1 / 2$, we have for any $\omega$,

$$
\left|\hat{u}_{2}(x, \omega)\right| \leq \frac{4 \nu}{a^{2}}\left|\hat{g}_{2}(\omega)\right| e^{\operatorname{Re} \lambda_{+}\left(x-L_{2}\right)} \leq C \nu\left|\hat{g}_{2}(\omega)\right| .
$$

By Parseval's identity, we obtain

$$
\left\|u_{2}(x, \cdot)\right\|_{L^{2}\left(\mathbb{R}_{+}\right)} \leq C \nu\left\|g_{2}\right\|_{L^{2}\left(\mathbb{R}_{+}\right)} .
$$


Modifying now $g_{2}$ to vanish in $[T+\epsilon, \infty)$, the solution in $(0, T)$ remains unaffected by causality and for any positive $\epsilon$,

$$
\left\|u_{2}(x, \cdot)\right\|_{L^{2}(0, T)} \leq C \nu\left\|g_{2}\right\|_{L^{2}(0, T+\epsilon)} .
$$

Since $\epsilon$ is arbitrary, we conclude that

$$
\left\|u_{2}(x, \cdot)\right\|_{L^{2}(0, T)} \leq C \nu\left\|g_{2}\right\|_{L^{2}(0, T)} .
$$

From (3.9) we also obtain for all $\omega$

$$
\left\|\hat{u}_{2}(\cdot, \omega)\right\|_{L_{x}^{2}}^{2} \leq \frac{C \nu^{2}}{\operatorname{Re} \lambda_{+}}\left|\hat{g}_{2}(\omega)\right|^{2} \leq C \nu^{3}\left|\hat{g}_{2}(\omega)\right|^{2} .
$$

We thus obtain

$$
\left\|u_{2}\right\|_{L_{x, t}^{2}} \leq C \nu^{\frac{3}{2}}\left\|g_{2}\right\|_{L_{t}^{2}}
$$

For the derivative in space, we compute

$$
\begin{aligned}
\partial_{x} \hat{u}_{2}(x, \omega) & =\hat{g}_{2}(\omega) \frac{\lambda_{+} e^{\lambda_{+}\left(x+L_{1}\right)}-\lambda_{-} e^{\lambda_{-}\left(x+L_{1}\right)}}{\nu \lambda_{+}^{2} e^{\lambda_{+}\left(L_{2}+L_{1}\right)}-\nu \lambda_{-}^{2} e^{\lambda_{-}\left(L_{2}+L_{1}\right)}} \\
& =\hat{g}_{2}(\omega) e^{\lambda_{+}\left(x-L_{2}\right)} \frac{\frac{\lambda_{-}}{\lambda_{+}} e^{-\left(\lambda_{+}-\lambda_{-}\right)\left(x+L_{1}\right)}-1}{\nu \lambda_{+}\left(\left(\frac{\lambda_{-}}{\lambda_{+}}\right)^{2} e^{-\left(\lambda_{+}-\lambda_{-}\right)\left(L_{2}+L_{1}\right)}-1\right)} .
\end{aligned}
$$

For small $\nu$, we therefore get as before

$$
\left|\partial_{x} \hat{u}_{2}(x, \omega)\right| \leq \frac{4}{\nu\left|\lambda_{+}\right|}\left|\hat{g}_{2}(\omega)\right| e^{\operatorname{Re} \lambda_{+}\left(x-L_{2}\right)} \leq \frac{4}{a}\left|\hat{g}_{2}(\omega)\right| e^{\operatorname{Re} \lambda_{+}\left(x-L_{2}\right)},
$$

which gives $\left\|\partial_{x} \hat{u}_{2}\right\|_{L^{2}(\Omega \times \mathbb{R})}^{2} \leq C \nu\left\|\hat{g}_{2}\right\|_{L^{2}(\mathbb{R})}^{2}$, and using the same arguments as before,

$$
\left\|\partial_{x} u_{2}\right\|_{L_{x, t}^{2}} \leq C \nu^{\frac{1}{2}}\left\|g_{2}\right\|_{L_{t}^{2}} .
$$

In the same way we find that

$$
\left\|\partial_{x}^{2} u_{2}\right\|_{L_{x, t}^{2}} \leq C \nu^{-\frac{1}{2}}\left\|g_{2}\right\|_{L_{t}^{2}}
$$

TheOREm 3.3. Let $a>0$. Then there exist positive constants $C$ and $\bar{\nu}$ such that for any $\nu \leq \bar{\nu}$, and for any set of data $h \in \mathcal{C}_{0}^{\infty}(\Omega), f \in \mathcal{C}_{0}^{\infty}(\Omega \times(0, T]), g_{1} \in \mathcal{C}_{0}^{\infty}((0, T])$ and $g_{2} \equiv 0$, if $U$ is the solution of the transport equation

$$
\mathcal{L}_{a} U=f \text { in } \Omega \times(0, T), \quad U(\cdot, 0)=h \quad U\left(-L_{1}, \cdot\right)=g_{1},
$$

then the solution $u$ of the advection-diffusion equation (3.1) satisfies the estimate

$$
\begin{aligned}
\left\|\partial_{t}^{k}(u-U)\right\|_{L_{x, t}^{2}}^{2} & +\left\|\partial_{t}^{k}(u-U)\left(L_{2}, \cdot\right)\right\|_{L_{t}^{2}}^{2}+\nu\left\|\partial_{t}^{k} \partial_{x}(u-U)\right\|_{L_{x, t}^{2}}^{2} \\
& +\nu^{2}\left\|\partial_{t}^{k} \partial_{x}^{2}(u-U)\right\|_{L_{x, t}^{2}}^{2} \leq C \nu^{2}\left\|\partial_{t}^{k} \partial_{x}^{2} U\right\|_{L_{x, t}^{2}}^{2}
\end{aligned}
$$

Hence $u$ also satisfies the estimate

$$
\begin{aligned}
\left\|\partial_{t}^{k} u\right\|_{L_{x, t}^{2}}^{2} & +\left\|\partial_{t}^{k} u\left(L_{2}, \cdot\right)\right\|_{L_{t}^{2}}^{2}+\left\|\partial_{t}^{k} \partial_{x} u\right\|_{L_{x, t}^{2}}^{2} \\
& +\left\|\partial_{t}^{k} \partial_{x}^{2} u\right\|_{L_{x, t}^{2}}^{2} \leq C\left(\|f\|_{H_{x, t}^{k+2}}^{2}+\|h\|_{H_{x}^{k+2}}^{2}+\left\|g_{1}\right\|_{H_{t}^{k+2}}^{2}\right) .
\end{aligned}
$$


Proof. Since the data is compactly supported, the compatibility conditions are automatically satisfied, so that the solutions of the parabolic and hyperbolic equations are in $\mathcal{C}_{0}^{\infty}(\Omega \times(0, T])$. The estimates (3.12) follow directly from Lemma 3.1] using that $u-U$ is solution of the advection-diffusion equation in $\Omega$ with right hand side $\nu \partial_{x}^{2} U$, and zero initial and boundary conditions on the left. The boundary condition on the right also vanishes, $\mathcal{L}_{a}(u-U)=-f\left(L_{2}, \cdot\right)=0$, since $f$ is compactly supported in $\Omega$. We define now $B_{k}=\|f\|_{H_{x, t}^{k}}^{2}+\|h\|_{H_{x}^{k}}^{2}+\left\|g_{1}\right\|_{H_{t}^{k}}^{2}$, and use for $U$ the hyperbolic estimates (2.15) in $\mathcal{O}=\Omega$,

$$
\left\|\partial_{t}^{k} U\right\|_{L_{x, t}^{2}}^{2}+\left\|\partial_{t}^{k} U\left(L_{2}, \cdot\right)\right\|_{L_{t}^{2}}^{2} \leq C B_{k} .
$$

Next, from the advection equation, we deduce that

$$
a \partial_{x} U=-\left(\partial_{t}+c\right) U+f, \quad \text { and } \quad a^{2} \partial_{x}^{2} U=\left(\partial_{t}+c\right)^{2} U+\left(a \partial_{x}-\left(\partial_{t}+c\right)\right) f,
$$

so that

$$
\left\|\partial_{t}^{k} \partial_{x} U\right\|_{L_{x, t}^{2}}^{2} \leq C B_{k+1}, \quad\left\|\partial_{t}^{k} \partial_{x}^{2} U\right\|_{L_{x, t}^{2}}^{2} \leq C B_{k+2},
$$

and from (3.12) we obtain

$$
\begin{aligned}
& \left\|\partial_{t}^{k}(u-U)\right\|_{L_{x, t}^{2}}^{2}+\left\|\partial_{t}^{k}(u-U)\left(L_{2}, \cdot\right)\right\|_{L_{t}^{2}}^{2} \leq C \nu^{2} B_{k+2}, \\
& \left\|\partial_{t}^{k} \partial_{x}(u-U)\right\|_{L_{x, t}^{2}}^{2} \leq C \nu B_{k+2}, \quad\left\|\partial_{t}^{k} \partial_{x}^{2}(u-U)\right\|_{L_{x, t}^{2}}^{2} \leq C B_{k+2} .
\end{aligned}
$$

Writing $u=u-U+U$ gives

$$
\begin{aligned}
& \left\|\partial_{t}^{k} u\right\|_{L_{x, t}^{2}}^{2}+\left\|\partial_{t}^{k} u\left(L_{2}, \cdot\right)\right\|_{L_{t}^{2}}^{2} \leq C\left(B_{k}+\nu^{2} B_{k+2}\right), \\
& \left\|\partial_{t}^{k} \partial_{x} u\right\|_{L_{x, t}^{2}}^{2} \leq C\left(B_{k+1}+\nu B_{k+2}\right), \quad\left\|\partial_{t}^{k} \partial_{x}^{2} u\right\|_{L_{x, t}^{2}}^{2} \leq C B_{k+2} .
\end{aligned}
$$

Therefore there is a new constant $C$ and $\bar{\nu}$ such that for $\nu \leq \bar{\nu}$, 3.13) holds.

We now present an improved estimate for the solution of the modified advection problem in $\Omega_{2}$.

TheOREM 3.4. Let $a>0$. Then there exist positive constants $C$ and $\bar{\nu}$ such that, for $\nu \leq \bar{\nu}$, and for any set $p$ compactly supported in $\bar{\Omega}_{2} \times(0, T]$, the solution $v$ of the initial boundary value problem with modified advection

$$
\left\{\begin{array}{rll}
\mathcal{L}_{m a} v & = & \text { in }\left(0, L_{2}\right) \times(0, T), \\
v\left(L_{2}, \cdot\right) & =0 & \text { on }(0, T) \\
v(\cdot, 0) & =0 & \text { in }\left(0, L_{2}\right)
\end{array}\right.
$$

satisfies the estimate

$$
\left\|\partial_{t}^{k} v(0, \cdot)\right\|_{L_{t}^{2}}^{2} \leq C \nu^{2}\left(\left\|\partial_{t}^{k} p(0, \cdot)\right\|_{L_{t}^{2}}^{2}+e^{-2 \frac{a L_{2}}{\nu}}\left\|\partial_{t}^{k} p\left(L_{2}, \cdot\right)\right\|_{L_{t}^{2}}^{2}+\nu\left\|\partial_{t}^{k} p\right\|_{H_{x, t}^{1}}^{2}\right) .
$$

Proof. We first extend $p$ by 0 on $(T,+\infty)$. As in Theorem 2.2, $v$ can be obtained using the method of characteristics,

$$
v(0, t)=\int_{\max \left(t-\frac{L_{2}}{a}, 0\right)}^{t} p(a(t-\sigma), \sigma) e^{-\tilde{c}(t-\sigma)} d \sigma,
$$


where $\tilde{c}=c+a^{2} / \nu$. Integrating by parts, and denoting by $d_{t}$ the characteristic derivative, i.e. $d_{t} \phi=\partial_{t} \phi-a \partial_{x} \phi$, we obtain

$$
\begin{aligned}
v(0, t)=\frac{1}{\tilde{c}}(\underbrace{p(0, t)}_{I} & -\underbrace{ \begin{cases}0 & \text { for } t<L_{2} / a \\
p\left(L_{2}, t-\frac{L_{2}}{a}\right) e^{-\tilde{c} \frac{L_{2}}{a}} & \text { for } t>L_{2} / a\end{cases} }_{I I} \\
& -\underbrace{\int_{\max \left(t-\frac{L_{2}}{a}, 0\right)}^{t} d_{t} p(a(t-\sigma), \sigma) e^{-\tilde{c}(t-\sigma)} d \sigma}_{I I I}) .
\end{aligned}
$$

The norm of the first term is $\|I\|_{L_{t}^{2}}=\|p(0, \cdot)\|_{L_{t}^{2}}$, and the norm of the second term can be estimated as

$$
\|I I\|_{L_{t}^{2}}^{2}=\int_{\frac{L_{2}}{a}}^{+\infty} p^{2}\left(L_{2}, t-\frac{L_{2}}{a}\right) e^{-2 \tilde{c} \frac{L_{2}}{a}} d t \leq e^{-2 \tilde{c} L_{2} / a}\left\|p\left(L_{2}, \cdot\right)\right\|_{L_{t}^{2}}^{2} .
$$

For the norm of the third term, we get

$$
\begin{aligned}
\|I I I\|_{L_{t}^{2}}^{2} & =\int_{0}^{\frac{L_{2}}{a}}\left(\int_{0}^{t} d_{t} p(a(t-\sigma), \sigma) e^{-\tilde{c}(t-\sigma)} d \sigma\right)^{2} d t \\
& +\int_{\frac{L_{2}}{a}}^{+\infty}\left(\int_{t-\frac{L_{2}}{a}}^{t} d_{t} p(a(t-\sigma), \sigma) e^{-\tilde{c}(t-\sigma)} d \sigma\right)^{2} d t
\end{aligned}
$$

and using the Cauchy-Schwarz inequality, we obtain

$$
\begin{aligned}
\|I I I\|_{L_{t}^{2}}^{2} & \leq \frac{1}{2 \tilde{c}}\left(\int_{0}^{\frac{L_{2}}{a}} \int_{0}^{t}\left(d_{t} p\right)^{2}(a(t-\sigma), \sigma) d \sigma d t+\int_{\frac{L_{2}}{a}}^{+\infty} \int_{t-\frac{L_{2}}{a}}^{t}\left(d_{t} p\right)^{2}(a(t-\sigma), \sigma) d \sigma d t\right) \\
& \leq \frac{1}{2 \tilde{c}} \int_{0}^{+\infty} \int_{0}^{L_{2}}\left(d_{t} p\right)^{2}(x, t) d x d t \leq \frac{1}{2 \tilde{c}}\|p\|_{H_{x, t}^{1}}^{2},
\end{aligned}
$$

which finally leads to the estimate

$$
\|v(0, \cdot)\|_{L_{t}^{2}}^{2} \leq \frac{C}{\tilde{c}^{2}}\left(\|p(0, \cdot)\|_{L_{t}^{2}}^{2}+e^{-2 \tilde{c} \frac{L_{2}}{a}}\left\|p\left(L_{2}, \cdot\right)\right\|_{L_{t}^{2}}^{2}+\frac{1}{2 \tilde{c}}\|p\|_{H_{x, t}^{1}}^{2}\right) .
$$

Since $\tilde{c} \geq \frac{a^{2}}{\nu}$, we get

$$
\|v(0, \cdot)\|_{L_{t}^{2}}^{2} \leq C \nu^{2}\left(\|p(0, \cdot)\|_{L_{t}^{2}}^{2}+e^{-2 \frac{a L_{2}}{\nu}}\left\|p\left(L_{2}, \cdot\right)\right\|_{L_{t}^{2}}^{2}+\nu\|p\|_{H_{x, t}^{1}}^{2}\right)
$$

on the enlarged time interval $(0,+\infty)$. Using that the extension is vanishing for $t \geq T+\epsilon$ gives the estimate (3.14) for $k=0$ for any $\epsilon$, and thus on $(0, T)$. Applying (3.14) to $\partial_{t}^{k} v$ gives then the general result.

Theorem 3.5. Assume that $a>0$, and let $B_{k}:=\|f\|_{H_{x, t}^{k}}^{2}+\|h\|_{H_{x}^{k}}^{2}+\left\|g_{1}\right\|_{H_{t}^{k}}^{2}$. Then there exist positive constants $C$ and $\bar{\nu}$ such that, for any data $h \in \mathcal{C}_{0}^{\infty}\left(\Omega_{1}\right), f \in$ $\mathcal{C}_{0}^{\infty}(\Omega \times(0, T]), g_{1} \in \mathcal{C}_{0}^{\infty}((0, T])$ and $g_{2} \equiv 0$, and for any initial guess $g_{a d}^{0} \in \mathcal{C}_{0}^{\infty}((0, T])$ and any $\nu \leq \bar{\nu}$, the approximation from the new algorithm (2.5) satisfies the error bounds

$$
\begin{array}{ll}
\left\|u-u_{a}^{1}\right\|_{L_{x, t}^{2}}^{2} \leq C\left(B_{2}+\left\|g_{a d}^{0}\right\|_{L_{t}^{2}}^{2}\right), & \left\|u-u_{a d}^{1}\right\|_{L_{x, t}^{2}}^{2} \leq C \nu^{5}\left(B_{5}+\left\|g_{a d}^{0}\right\|_{H_{t}^{3}}^{2}\right) \\
\left\|u-u_{a}^{2}\right\|_{L_{x, t}^{2}}^{2} \leq C \nu^{2}\left(B_{5}+\nu^{2}\left\|g_{a d}^{0}\right\|_{H_{t}^{3}}^{2}\right), & \left\|u-u_{a d}^{2}\right\|_{L_{x, t}^{2}}^{2} \leq C \nu^{8}\left(B_{8}+\nu\left\|g_{a d}^{0}\right\|_{H_{t}^{6}}^{2}\right) .
\end{array}
$$


Proof. We start with the proof for the first iteration of the new algorithm (2.5), with the initial guess $g_{a d}^{0}$, which gives $e_{a d}^{0}=g_{a d}^{0}-u(0, \cdot)$ in the algorithm (3.3) satisfied by the errors:

Advection: The error $e_{a}^{1}$ is solution of an advection equation in $\Omega_{2}$ with right hand side $-\nu \partial_{x}^{2} u$. Since the initial conditions vanish as described in (3.4), the hyperbolic estimate in Theorem 2.2 gives

$$
\left\|\partial_{t}^{k} e_{a}^{1}\right\|_{L_{x, t}^{2}}^{2}+\left\|\partial_{t}^{k} e_{a}^{1}\left(L_{2}, \cdot\right)\right\|_{L_{t}^{2}}^{2} \leq C\left(\nu^{2}\left\|\partial_{t}^{k} \partial_{x}^{2} u\right\|_{L_{x, t}^{2}}^{2}+\left\|e_{a d}^{0}\right\|_{H_{t}^{k}}^{2}\right) .
$$

We bound $\left\|e_{a d}^{0}\right\|_{H_{t}^{k}}$ by $\left\|g_{a d}^{0}\right\|_{H_{t}^{k}}+\|u(0, \cdot)\|_{H_{t}^{k}}$. From (13.13), for small $\nu,\left\|\partial_{t}^{k} u(0, \cdot)\right\|_{L_{x, t}^{2}}^{2}$ (by the trace theorem) and $\left\|\partial_{t}^{k} \partial_{x}^{2} u\right\|_{L_{x, t}^{2}}^{2}$ are bounded by $C B_{k+2}$, which gives

$$
\left\|\partial_{t}^{k} e_{a}^{1}\right\|_{L_{x, t}^{2}}^{2}+\left\|\partial_{t}^{k} e_{a}^{1}\left(L_{2}, \cdot\right)\right\|_{L_{t}^{2}}^{2} \leq C\left(\nu^{2} B_{k+2}+\left\|e_{a d}^{0}\right\|_{H_{t}^{k}}^{2}\right) \leq C\left(\nu^{2} B_{k+2}+\left\|g_{a d}^{0}\right\|_{H_{t}^{k}}^{2}+B_{k+2}\right) .
$$

This equation gives for $k=0$ and small $\nu$ the first estimate in (3.16).

Modified advection: Let $p_{a}^{1}(0, \cdot):=\left(\partial_{t}+c\right)^{2} e_{a}^{1}(0, \cdot)=\left(\partial_{t}+c\right)^{2} e_{a d}^{0}$. We estimate $\partial_{t}^{k} e_{m a}^{1}$ at $x=0$ using Theorem 3.4

$$
\begin{aligned}
\left\|\partial_{t}^{k} e_{m a}^{1}(0, \cdot)\right\|_{L_{t}^{2}}^{2} & \leq C \nu^{2}\left(\left\|\partial_{t}^{k} p_{a}^{1}(0, \cdot)\right\|_{L_{t}^{2}}^{2}+e^{-2 \frac{a L_{2}}{\nu}}\left\|\partial_{t}^{k} p_{a}^{1}\left(L_{2}, \cdot\right)\right\|_{L_{t}^{2}}^{2}+\nu\left\|\partial_{t}^{k} p_{a}^{1}\right\|_{H_{x, t}^{1}}^{2}\right) \\
& \leq C \nu^{2}\left(\left\|e_{a d}^{0}\right\|_{H_{t}^{k+2}}^{2}+e^{-2 \frac{a L_{2}}{\nu}}\left\|\partial_{t}^{k} p_{a}^{1}\left(L_{2}, \cdot\right)\right\|_{L_{t}^{2}}^{2}+\nu\left\|\partial_{t}^{k} p_{a}^{1}\right\|_{H_{x, t}^{1}}^{2}\right) .
\end{aligned}
$$

For the last term on the right, we obtain

$$
\begin{aligned}
\left\|\partial_{t}^{k} p_{a}^{1}\right\|_{H_{x, t}^{1}}^{2} & =\left\|\partial_{t}^{k} p_{a}^{1}\right\|_{L_{x, t}^{2}}^{2}+\left\|\partial_{x} \partial_{t}^{k} p_{a}^{1}\right\|_{L_{x, t}^{2}}^{2}+\left\|\partial_{t}^{k+1} p_{a}^{1}\right\|_{L_{x, t}^{2}}^{2} \\
& \leq C\left(\left\|p_{a}^{1}\right\|_{H^{k+1}\left(0, T ; L_{x}^{2}\right)}^{2}+\left\|\partial_{x} p_{a}^{1}\right\|_{H^{k}\left(0, T ; L_{x}^{2}\right)}^{2}\right) \\
& \leq C\left(\left\|e_{a}^{1}\right\|_{H^{k+3}\left(0, T ; L_{x}^{2}\right)}^{2}+\left\|\partial_{x} e_{a}^{1}\right\|_{H^{k+2}\left(0, T ; L_{x}^{2}\right)}^{2}\right) .
\end{aligned}
$$

Since $\mathcal{L}_{a} e_{a}^{1}=-\nu \partial_{x}^{2} u$, we get $\partial_{x} e_{a}^{1}=-\frac{1}{a}\left(\nu \partial_{x}^{2} u+\left(\partial_{t}+c\right) e_{a}^{1}\right)$, and hence $\left\|\partial_{x} e_{a}^{1}\right\|_{H^{k+2}\left(0, T ; L_{x}^{2}\right)} \leq$ $C\left(\nu\left\|\partial_{x}^{2} u\right\|_{H^{k+2}\left(0, T ; L_{x}^{2}\right)}+\left\|e_{a}^{1}\right\|_{H^{k+3}\left(0, T ; L_{x}^{2}\right)}\right)$. Using (3.18), we finally obtain

$$
\left\|\partial_{t}^{k} p_{a}^{1}\right\|_{H_{x, t}^{1}}^{2} \leq C\left(\nu^{2} B_{k+5}+\left\|e_{a d}^{0}\right\|_{H_{t}^{k+3}}^{2}+\nu^{2}\left\|\partial_{x}^{2} u\right\|_{H^{k+2}\left(0, T ; L_{x}^{2}\right)}^{2}\right) .
$$

We use now (3.13) which gives $\left\|\partial_{x}^{2} u\right\|_{H^{k+2}\left(0, T ; L_{x}^{2}\right)}^{2} \leq C B_{k+4}$ and return to (3.19). From the hyperbolic estimate (3.18), we see that $\left\|\partial_{t}^{k} e_{a}^{1}\left(L_{2}, \cdot\right)\right\|_{L_{t}^{2}}^{2}$ and $\left\|\partial_{t}^{k} e_{a}^{1}\right\|_{L_{x, t}^{2}}^{2}$ are bounded by the same quantity, and hence we can also use the same bound for $e^{-2 \frac{a L_{2}}{\nu}}\left\|\partial_{t}^{k} p_{a}^{1}\left(L_{2}, \cdot\right)\right\|_{L_{t}^{2}}^{2}$ and $\nu\left\|\partial_{t}^{k} p_{a}^{1}\right\|_{H_{x, t}^{1}}^{2}$,

$$
\left\|\partial_{t}^{k} e_{m a}^{1}(0, \cdot)\right\|_{L_{t}^{2}}^{2} \leq C\left(\nu^{2}\left\|e_{a d}^{0}\right\|_{H_{t}^{k+3}}^{2}+\nu^{5} B_{k+5}\right) .
$$

Advection-diffusion: $e_{a d}^{1}$ is solution of the advection-diffusion equation with non-zero data only on the right. Therefore, applying Lemma 3.2 in $\Omega_{1}$ with $g_{2}=$ $e_{m a}^{1}(0, \cdot)$, we obtain

$$
\left\|e_{a d}^{1}\right\|_{L_{x, t}^{2}}^{2} \leq C \nu^{3}\left\|e_{m a}^{1}(0, \cdot)\right\|_{L_{t}^{2}}^{2} .
$$

Using (3.20) with $k=0$ for the last term, we obtain

$$
\left\|e_{a d}^{1}\right\|_{L_{x, t}^{2}}^{2} \leq C \nu^{5}\left(\left\|e_{a d}^{0}\right\|_{H_{t}^{3}}^{2}+\nu^{2} B_{5}\right) \leq C \nu^{5}\left(B_{5}+\left\|g_{a d}^{0}\right\|_{H_{t}^{3}}^{2}\right) .
$$


This equation gives the second estimate in (3.16), but we will also need to estimate the value of $e_{a d}^{1}$ at $x=0$. Using (3.7) we get

$$
\left\|\partial_{t}^{k} e_{a d}^{1}(0, \cdot)\right\|_{L_{t}^{2}}^{2} \leq C \nu^{2}\left\|\partial_{t}^{k} e_{m a}^{1}(0, \cdot)\right\|_{L_{t}^{2}}^{2},
$$

which gives by (3.20) again

$$
\left\|\partial_{t}^{k} e_{a d}^{1}(0, \cdot)\right\|_{L_{t}^{2}}^{2} \leq C \nu^{4}\left(\nu^{3} B_{k+5}+\left\|e_{a d}^{0}\right\|_{H_{t}^{k+3}}^{2}\right) .
$$

In particular, we have

$$
\left\|\mathcal{R} e_{a d}^{1}(0, \cdot)\right\|_{L_{t}^{2}}^{2} \leq C \nu^{4}\left(\nu^{3} B_{7}+\left\|e_{a d}^{0}\right\|_{H_{t}^{5}}^{2}\right) .
$$

We now prove the error estimates for the second iteration:

Advection: We again use the hyperbolic estimates for $e_{a}^{2}$. Since the initial values are also vanishing, we obtain as in (3.18) the estimate

$$
\left\|\partial_{t}^{k} e_{a}^{2}\right\|_{L_{x, t}^{2}}^{2}+\left\|\partial_{t}^{k} e_{a}^{2}\left(L_{2}, \cdot\right)\right\|_{L_{t}^{2}}^{2} \leq C\left(\nu^{2} B_{k+2}+\left\|e_{a d}^{1}(0, \cdot)\right\|_{H_{t}^{k}}^{2}\right) .
$$

Inserting (3.22) we get

$$
\begin{aligned}
\left\|\partial_{t}^{k} e_{a}^{2}\left(L_{2}, \cdot\right)\right\|_{L_{t}^{2}}^{2}+\left\|\partial_{t}^{k} e_{a}^{2}\right\|_{L_{x, t}^{2}}^{2} & \leq C\left(\nu^{2} B_{k+2}+\nu^{4}\left(\nu^{3} B_{k+5}+\left\|e_{a d}^{0}\right\|_{H_{t}^{k+3}}^{2}\right)\right) \\
& \leq C \nu^{2}\left(B_{k+5}+\nu^{2}\left\|e_{a d}^{0}\right\|_{H_{t}^{k+3}}^{2}\right) \\
& \leq C \nu^{2}\left(B_{k+5}+\nu^{2}\left\|g_{a d}^{0}\right\|_{H_{t}^{k+3}}^{2}+\nu^{2} B_{k+5}\right) .
\end{aligned}
$$

The last estimate with $k=0$ gives the first result in (3.17).

Modified advection: Defining $p_{a}^{2}:=\mathcal{R} e_{a}^{2}=\left(\partial_{t}+c\right)^{2} e_{a}^{2}$, we obtain using (3.25)

$$
\left\|\partial_{t}^{k} p_{a}^{2}\left(L_{2}, \cdot\right)\right\|_{L_{t}^{2}}^{2}+\left\|\partial_{t}^{k} p_{a}^{2}\right\|_{L_{x, t}^{2}}^{2} \leq C \nu^{2}\left(B_{k+7}+\left\|e_{a d}^{0}\right\|_{H_{t}^{k+5}}^{2}\right) .
$$

We estimate $e_{m a}^{2}$ at $x=0$ by Theorem 3.4

$$
\left\|e_{m a}^{2}(0, \cdot)\right\|_{L_{t}^{2}}^{2} \leq C \nu^{2}\left(\left\|p_{a}^{2}(0, \cdot)\right\|_{L_{t}^{2}}^{2}+e^{-2 \frac{a L_{2}}{\nu}}\left\|p_{a}^{2}\left(L_{2}, \cdot\right)\right\|_{L_{t}^{2}}^{2}+\nu\left\|p_{a}^{2}\right\|_{H_{x, t}^{1}}^{2}\right) .
$$

As in the first step, the term at the boundary $x=L_{2}$ is absorbed in the volume term, and $p_{a}^{2}(0, \cdot)=\mathcal{R} e_{a d}^{1}(0, \cdot)$, which can be estimated by (3.23). To estimate the term $\left\|p_{a}^{2}\right\|_{H_{x, t}^{1}}$, we proceed as in the first iteration, to obtain

$$
\begin{aligned}
\left\|p_{a}^{2}\right\|_{H_{x, t}^{1}}^{2} & \leq C\left(\left\|e_{a}^{2}\right\|_{H^{3}\left(0, T ; L_{x}^{2}\right)}^{2}+\nu^{2}\left\|\partial_{x}^{2} u\right\|_{H^{2}\left(0, T ; L_{x}^{2}\right)}^{2}\right) \\
& \leq C\left(\left\|e_{a}^{2}\right\|_{H^{3}\left(0, T ; L_{x}^{2}\right)}^{2}+\nu^{2} B_{4}\right) \\
& \leq C \nu^{2}\left(B_{8}+\nu^{2}\left\|e_{a d}^{0}\right\|_{H_{t}^{6}}^{2}\right) .
\end{aligned}
$$

Inserting (3.23) and (3.28) into (3.27) we get

$$
\begin{aligned}
\left\|e_{m a}^{2}(0, \cdot)\right\|_{L_{t}^{2}}^{2} & \leq C \nu^{2}\left(\nu^{4}\left(\nu^{3} B_{7}+\left\|e_{a d}^{0}\right\|_{H_{t}^{5}}^{2}\right)+\nu^{3}\left(B_{8}+\nu^{2}\left\|e_{a d}^{0}\right\|_{H_{t}^{6}}^{2}\right)\right) \\
& \leq C \nu^{5}\left(B_{8}+\nu\left\|e_{a d}^{0}\right\|_{H_{t}^{6}}^{2}\right) .
\end{aligned}
$$

Advection-diffusion: $e_{a d}^{2}$ is solution of the advection-diffusion equation with data only on the right. Therefore, applying Lemma 3.2 in $\Omega_{1}$ with $g_{2}=e_{m a}^{2}(0, \cdot)$, we obtain

$$
\left\|e_{a d}^{2}\right\|_{L_{x, t}^{2}}^{2} \leq C \nu^{3}\left\|e_{m a}^{2}(0, \cdot)\right\|_{L_{t}^{2}}^{2} \leq C \nu^{8}\left(B_{8}+\nu\left\|e_{a d}^{0}\right\|_{H_{t}^{6}}^{2}\right)
$$


4. Properties of the factorization algorithm for negative advection. We consider now the advection-diffusion equation for $a<0$ in $\Omega=\left(-L_{1}, L_{2}\right)$ with Dirichlet boundary conditions on both sides,

$$
\begin{aligned}
\mathcal{L}_{a d} u:=\partial_{t} u-\nu \partial_{x x} u+a \partial_{x} u+c u & =f & & \text { in } \Omega \times(0, T), \\
u\left(-L_{1}, \cdot\right) & =g_{1} & & \text { in }(0, T), \\
u\left(L_{2}, \cdot\right) & =g_{2} & & \text { in }(0, T), \\
u(\cdot, 0) & =h & & \text { in } \Omega .
\end{aligned}
$$

We suppose again that $f$ and $\left(g_{1}, g_{2}\right)$ are compactly supported in $(0, T]$, and that $h$ is compactly supported in $\Omega_{1}=\left(-L_{1}, 0\right)$, and that for each $t$ the function $f(\cdot, t)$ is compactly supported in $\Omega$.

4.1. Well-posedness of the factorization algorithm. For $u_{a}^{1}$, suppose that $f \in H^{3+\frac{3}{4}}(\Omega \times(0, T)), g_{2} \in H^{3+\frac{3}{4}}(0, T), h \in H^{3+\frac{3}{4}}(\Omega)$, and that the compatibility conditions (2.14) are satisfied. Then we have a unique solution $u_{a}^{1}$ in $W^{3+\frac{3}{4}}\left(\Omega_{2} \times\right.$ $(0, T))$. For $u_{a}^{2}$, using the previous result, we have $f-\mathcal{R} u_{a}^{1} \in H^{1+\frac{3}{4}}\left(\Omega_{2} \times(0, T)\right)$, and $\mathcal{L}_{m a} u_{a}^{1} \in H^{2+\frac{3}{4}}\left(\Omega_{2} \times(0, T)\right)$. Therefore the traces at $x=L_{2}$ and $t=0$ are in $H^{1+\frac{3}{4}}$ and compatible. Thus (2.6) defines a unique $u_{a}^{2}$ in $H^{1+\frac{3}{4}}\left(\Omega_{2} \times(0, T)\right)$. Furthermore $u_{a}^{2}(0, \cdot) \in H^{\frac{5}{4}}(0, T)$. For $u_{a d}$, Theorem 2.3 applies with $\gamma=\frac{5}{4}$, and (2.6) defines a unique $u_{a d}$ in $H^{\frac{9}{2}, \frac{9}{4}}\left(\Omega_{1} \times(0, T)\right)$. Finally for $u$, using the regularity assumptions above, $u \in H^{2(\gamma+1), \gamma+1}(\Omega \times(0, T))$ with $\gamma=\frac{11}{8}$.

4.2. Error estimates for the factorization algorithm. We need a further lemma in order to obtain our asymptotic estimates.

Lemma 4.1. Suppose $a<0$, and let $g \in L^{2}(0, T)$. Then there exists a constant $C>0$, such that for all $\nu>0$ the solution $v$ of

$$
\begin{aligned}
\mathcal{L}_{a d} v & =0 & & \text { in } \Omega_{1} \times(0, T), \\
v\left(-L_{1}, \cdot\right) & =0 & & \text { on }(0, T), \\
\left(\partial_{t}-a \partial_{x}+\left(\frac{a^{2}}{\nu}+c\right)\right) v(0, \cdot) & =g & & \text { on }(0, T), \\
v(\cdot, 0) & =0 & & \text { in } \Omega_{1},
\end{aligned}
$$

satisfies the a priori estimate

$$
\|v\|_{L_{x, t}^{2}}^{2} \leq C \nu^{2}\|g\|_{L_{t}^{2}}^{2}
$$

Proof. Multiplying the equation by $v$, integrating on $\left(-L_{1}, 0\right)$ and using the boundary condition at $x=-L_{1}$ yields

$$
\frac{1}{2} \frac{d}{d t}\|v(\cdot, t)\|_{L_{x}^{2}}^{2}-\frac{|a|}{2} v^{2}(0, t)+\nu\left\|\partial_{x} v(\cdot, t)\right\|_{L_{x}^{2}}^{2}-\nu \partial_{x} v(0, t) v(0, t)+c\|v(\cdot, t)\|_{L_{x}^{2}}^{2}=0 .
$$

Inserting the boundary condition at $x=0$ we obtain

$$
\begin{aligned}
\frac{1}{2} \frac{d}{d t}\left(\|v(\cdot, t)\|_{L_{x}^{2}}^{2}+\frac{\nu}{|a|} v^{2}(0, t)\right)+\left(\frac{|a|}{2}+\frac{\nu c}{|a|}\right) v^{2}(0, t) \\
+\nu\left\|\partial_{x} v(\cdot, t)\right\|_{L_{x}^{2}}^{2}+c\|v(\cdot, t)\|_{L_{x}^{2}}^{2}=\frac{\nu}{|a|} g(t) v(0, t) .
\end{aligned}
$$

Using the inequality $\nu|g(t) v(0, t)| /|a| \leq \frac{\nu^{2}}{|a|^{3}} g^{2}(t)+\frac{|a|}{4} v^{2}(0, t)$ and integrating on the time interval $(0, T)$ gives for all $t \in(0, T)$

$$
c\|v\|_{L_{x, t}^{2}}^{2} \leq \frac{\nu^{2}}{|a|^{3}} \int_{0}^{t} g^{2}(\tau) d \tau
$$


We can now prove our main theorem for negative advection.

THEOREM 4.2. Suppose $a<0$. Then there are positive constants $C$ and $\bar{\nu}$ such that for any $h \in \mathcal{C}_{0}^{\infty}\left(\Omega_{1}\right), f \in \mathcal{C}_{0}^{\infty}(\Omega \times(0, T]), g_{1}, g_{2} \in \mathcal{C}_{0}^{\infty}((0, T])$, and for any $\nu \leq \bar{\nu}$, the solution obtained by the new factorization algorithm (2.6) satisfies the estimates

$$
\begin{aligned}
\left\|u-u_{a}^{1}\right\|_{L_{x, t}^{2}} & \leq C \nu\left\|\partial_{x}^{2} u\right\|_{L_{x, t}^{2}}, \\
\left\|u-u_{a d}\right\|_{L_{x, t}^{2}} & \leq C \nu^{2}\left(\|u\|_{H_{x, t}^{2,2}}+\|f(\cdot, 0)\|_{H_{x}^{2}}+\|h\|_{H_{x}^{4}}+\left\|\partial_{x}^{2} u\left(L_{2}, \cdot\right)\right\|_{L_{t}^{2}},\right.
\end{aligned}
$$

which implies that

$$
\left\|u-u_{a}^{1}\right\|_{L_{x, t}^{2}} \lesssim \nu, \quad\left\|u-u_{a d}\right\|_{L_{x, t}^{2}} \lesssim \nu^{2}
$$

Proof. We define the errors $e_{a}^{1}:=u_{a}^{1}-u, e_{a d}:=u_{a d}-u$, and $e_{a}^{2}:=u_{a}^{2}-\mathcal{L}_{m a} u$. Since $\mathcal{L}_{m a} u(\cdot, 0)=f(\cdot, 0)-2 a d_{x} h+a^{2} h / \nu+\nu d_{x}^{2} h$, the equations for the error are

$$
\left\{\begin{array} { l } 
{ \mathcal { L } _ { a } e _ { a } ^ { 1 } = - \nu \partial _ { x } ^ { 2 } u \text { in } \Omega _ { 2 } , } \\
{ e _ { a } ^ { 1 } ( L _ { 2 } , \cdot ) = 0 , } \\
{ e _ { a } ^ { 1 } ( \cdot , 0 ) = 0 , }
\end{array} \quad \left\{\begin{array} { l } 
{ \mathcal { L } _ { a } e _ { a } ^ { 2 } = \mathcal { R } e _ { a } ^ { 1 } \text { in } \Omega _ { 2 } , } \\
{ e _ { a } ^ { 2 } ( L _ { 2 } , \cdot ) = \mathcal { L } _ { m a } e _ { a } ^ { 1 } ( L _ { 2 } , \cdot ) , } \\
{ e _ { a } ^ { 2 } ( \cdot , 0 ) = - \nu d _ { x } ^ { 2 } h , }
\end{array} \quad \left\{\begin{array}{l}
\mathcal{L}_{a d} e_{a d}=0 \text { in } \Omega_{1}, \\
e_{a d}\left(-L_{1}, \cdot\right)=0, \\
\mathcal{L}_{m a} e_{a d}(0, \cdot)=e_{a}^{2}(0, \cdot), \\
e_{a d}(\cdot, 0)=0 .
\end{array}\right.\right.\right.
$$

We now analyze each of the three solves separately:

First advection equation in $\Omega_{2}$ : With Theorem 2.2, we find that the error $e_{a}^{1}$ satisfies for $k=0,1$ and 2 the estimate

$$
\left\|\partial_{t}^{k} e_{a}^{1}\right\|_{L_{x, t}^{2}}^{2}+|a|\left\|\partial_{t}^{k} e_{a}^{1}(0, \cdot)\right\|_{L_{t}^{2}}^{2} \leq C\left(\nu^{2}\left\|\partial_{t}^{k} \partial_{x}^{2} u\right\|_{L_{x, t}^{2}}^{2}+\left\|\partial_{t}^{k} e_{a}^{1}(\cdot, 0)\right\|_{L_{x}^{2}}^{2}\right) .
$$

The case $k=0$ yields the first result of the theorem. We further compute

$$
\partial_{t} e_{a}^{1}(\cdot, 0)=-\nu d_{x}^{2} h, \quad \partial_{t}^{2} e_{a}^{1}(\cdot, 0)=\nu\left(a d_{x}+c\right) d_{x}^{2} h-\nu d_{x}^{2} \partial_{t} u(\cdot, 0),
$$

with $\partial_{t} u(\cdot, 0)=f(\cdot, 0)-\left(a d_{x} h+c h-\nu d_{x}^{2} h\right)$, so that

$$
\left\|\partial_{t} e_{a}^{1}(\cdot, 0)\right\|_{L_{x}^{2}}^{2} \leq \nu^{2}\|h\|_{H_{x}^{2}}, \quad\left\|\partial_{t}^{2} e_{a}^{1}(\cdot, 0)\right\|^{2} \leq \nu^{2}\left(\|f(\cdot, 0)\|_{H_{x}^{2}}^{2}+\|h\|_{H_{x}^{4}}^{2}\right) .
$$

We thus obtain for $\mathcal{R} e_{a}^{1}=\left(c+\partial_{t}\right)^{2} e_{a}^{1}$ the estimate

$$
\left\|\mathcal{R} e_{a}^{1}\right\|_{L_{x, t}^{2}}^{2} \leq C \nu^{2}\left(\|u\|_{H_{x, t}^{2,2}}^{2}+\|f(\cdot, 0)\|_{H_{x}^{2}}^{2}+\|h\|_{H_{x}^{4}}^{2}\right) .
$$

Second advection equation in $\Omega_{2}$ : Using again Theorem 2.2, we obtain the estimate

$$
\left\|e_{a}^{2}(0, \cdot)\right\|_{L_{t}^{2}}^{2} \leq C\left(\left\|\mathcal{R} e_{a}^{1}\right\|_{L_{x, t}^{2}}^{2}+\nu^{2}\|h\|_{H_{x}^{2}}^{2}+\left\|\mathcal{L}_{m a} e_{a}^{1}\left(L_{2}, \cdot\right)\right\|_{L_{t}^{2}}^{2}\right) .
$$

To evaluate $\mathcal{L}_{m a} e_{a}^{1}\left(L_{2}, \cdot\right)$, we observe that $u\left(L_{2}, \cdot\right)=u_{a}\left(L_{2}, \cdot\right)$, so that we have $\mathcal{L}_{m a} e_{a}^{1}\left(L_{2}, \cdot\right)=-a \partial_{x} e_{a}^{1}\left(L_{2}, \cdot\right)=\nu \partial_{x}^{2} u\left(L_{2}, \cdot\right)$. Therefore, using (4.6), we get

$$
\left\|e_{a}^{2}(0, \cdot)\right\|_{L_{t}^{2}}^{2} \leq C \nu^{2}\left(\|u\|_{H_{x, t}^{2,2}}^{2}+\|f(\cdot, 0)\|_{H_{x}^{2}}^{2}+\|h\|_{H_{x}^{4}}^{2}+\left\|\partial_{x}^{2} u\left(L_{2}, \cdot\right)\right\|_{L_{t}^{2}}^{2}\right) .
$$

Advection-diffusion equation in $\Omega_{1}$ : With Lemma 4.1 we obtain

$$
\left\|e_{a d}\right\|_{L_{x, t}^{2}}^{2} \leq C \nu^{2}\left\|e_{a}^{2}(0, \cdot)\right\|_{L_{t}^{2}}^{2} .
$$



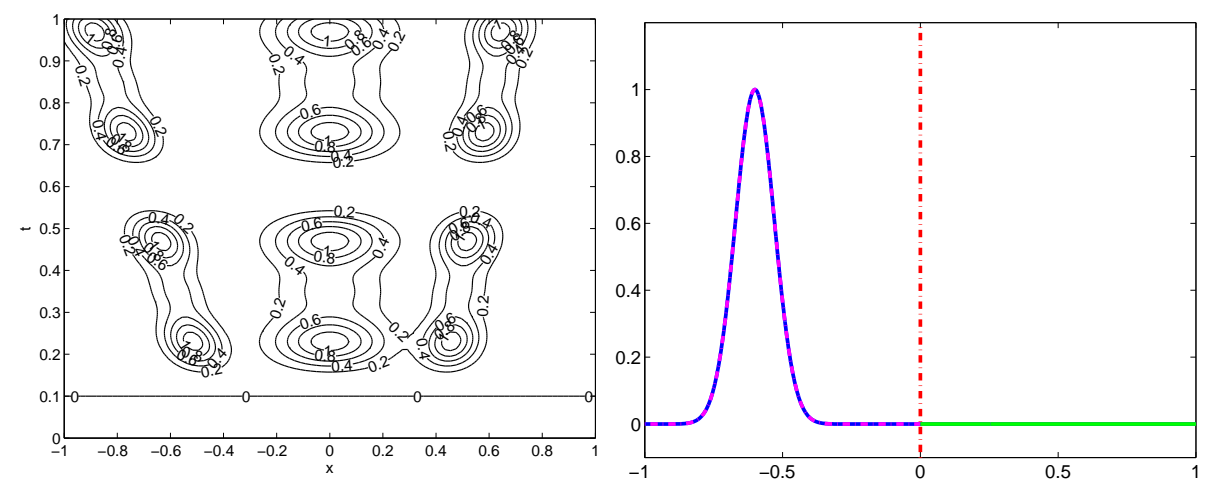

FIG. 5.1. Left: contour plot for the right hand side in space and time. Right: initial condition $a>0$

We can thus conclude using (4.7).

It remains to estimates $\left\|\partial_{x}^{2} u\left(L_{2}, \cdot\right)\right\|_{L_{t}^{2}}$ and $\left\|\partial_{x}^{2} u\right\|_{L^{2}\left(\Omega_{2} \times(0, T)\right)}$. If the data is compactly supported, there is only one boundary layer, at $x=-L_{1}$, and (see [31])

$$
u(t, x)=U(t, x)+e^{a\left(x+L_{1}\right) / \nu} U(t, 0)+\mathcal{O}(\nu), \quad(\text { note } a<0) .
$$

Here, $U$ is the solution of the advection equation in $\Omega$ with data $g_{2}$ at $x=L_{2}$. The norm of $\partial_{x x} u$, though not bounded in the entire interval $\Omega$, is bounded in $\Omega_{2}$, since

$$
\left\|d_{x}^{2} e^{a\left(x+L_{1}\right) / \nu}\right\|_{L^{2}\left(\Omega_{2}\right)}^{2}=\frac{|a|^{3}}{2 \nu^{3}}\left(e^{2 a L_{1} / \nu}-e^{2 a\left(L_{2}+L_{1}\right) / \nu}\right) \sim \frac{|a|^{3}}{2 \nu^{3}} e^{2 a L_{1} / \nu},
$$

which tends to zero as $\nu$ goes to zero, because $a<0$. Similarly the value at $L_{2}$ is bounded.

5. Numerical Experiments. We use a Crank-Nicolson scheme for the advectiondiffusion equation and an implicit upwind scheme for the advection equation. We discretize $\Omega:=(-1,1)$ with $N=64000$ points, which leads to a spatial step $\Delta x=$ $3.125 \times 10^{-5}$ and the time step $\Delta t=\Delta x$. We choose $c=1, g_{1} \equiv g_{2} \equiv 0, T=1$ and the right hand side, shown in Figure 5.1 on the left, is

$$
\begin{aligned}
f(x, t) & =f_{1}(t) f_{2}(x, t), \\
f_{1}(t) & =\left(\sin ^{4}\left(4 \pi\left(t-t_{0}\right)\right)+\sin ^{4}\left(2 \pi\left(t-t_{0}\right)\right) / 2\right) \chi_{t>t_{0}}, \quad t_{0}=0.1, \\
f_{2}(x, t) & =e^{-100 x^{2} / 4}+e^{-100(x-t / 4-0.4)^{2}}+e^{-100(x+t / 2+0.4)^{2}} .
\end{aligned}
$$

5.1. Positive advection. We choose $a=1$, with the initial condition, shown in Figure 5.1 on the right,

$$
u_{0}(x)=e^{-100\left(x-x_{0}\right)^{2}}, \text { with } x_{0}=-0.6 .
$$

Figure 5.2 shows first snapshots in time of the right hand side, and then of the viscous solution (3.1) and the solution obtained by the factorization algorithm (2.5) after one and two iterations when $\nu=10^{-3}$. We see that in the first iteration the solution $u_{a d}^{1}$ is very close to the viscous solution. This solution is improved with the second iteration when $u_{a}$ is also improved. 

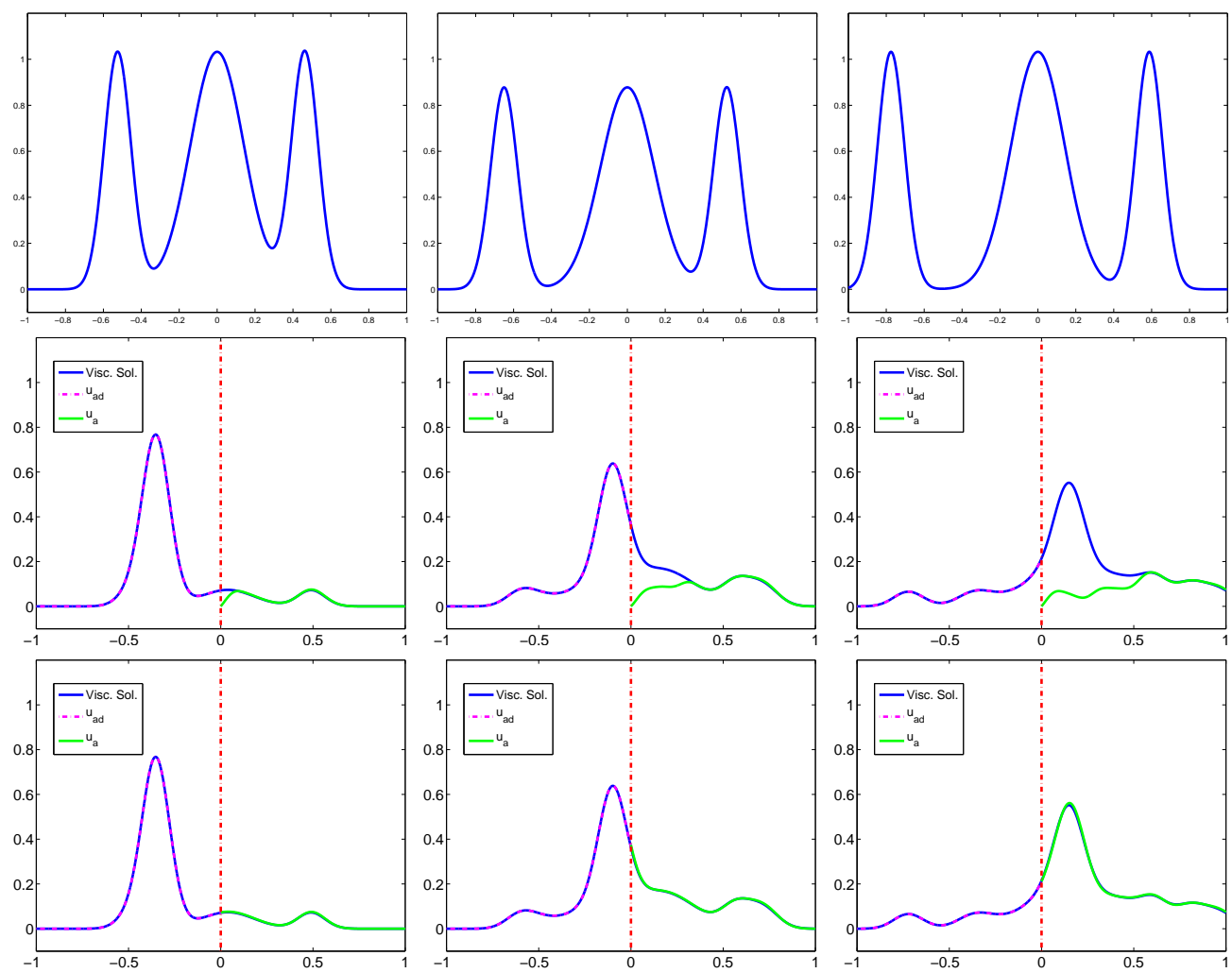

FIG. 5.2. From left to right: snapshots at time $t=0.25,0.5$ and 0.75. First line: right hand side. Second line: solution of Algorithm 2.5) at iteration $k=1$. Third line: solution of Algorithm (2.5) at iteration $k=2$

Figure 5.3 shows the $L^{2}$ space-time error as a function of the viscosity becoming small for the factorization algorithm (2.5) and gives a comparison to algorithms from the literature. These algorithms solve an advection-diffusion equation $\mathcal{L}_{a d} u_{a d}=f$ in $\Omega_{1}$ and an advection equation $\mathcal{L}_{a} u_{a}=f$ in $\Omega_{2}$, and use for $a>0$ either nonvariational transmission conditions $\partial_{x} u_{a d}(0, \cdot)=\partial_{x} u_{a}(0, \cdot)$ and $u_{a d}(0, \cdot)=u_{a}(0, \cdot)$, see [13, 23], or variational transmission conditions $\nu \partial_{x} u_{a d}(0, \cdot)=0$ and $u_{a d}(0, \cdot)=u_{a}(0, \cdot)$, see 23,24 . We see that the variational transmission conditions do not need an iteration in this case, one can first solve advection-diffusion, and then advection. The error is however $\mathcal{O}\left(\nu^{\frac{3}{2}}\right)$ in the viscous region $\Omega_{1}$. With only one iteration of the factorization algorithm, the error is $\mathcal{O}\left(\nu^{\frac{5}{2}}\right)$, and with two iterations we get $\mathcal{O}\left(\nu^{4}\right)$, both corresponding to our theoretical results in Theorem 3.5. The non-variational transmission conditions also give an error $\mathcal{O}\left(\nu^{\frac{5}{2}}\right)$, as good as with one iteration of the factorization algorithm, but one needs to iterate and choosing a good relaxation parameter to ensure convergence is not easy; we chose heuristically $\theta=\frac{1}{450 \sqrt{\nu}}$ in our computations. In the inviscid subregion $\Omega_{2}$, the error of all methods is $\mathcal{O}(\nu)$, only the initialization step in the factorization algorithm has an error of $\mathcal{O}(1)$, as predicted by Theorem 3.5 . 

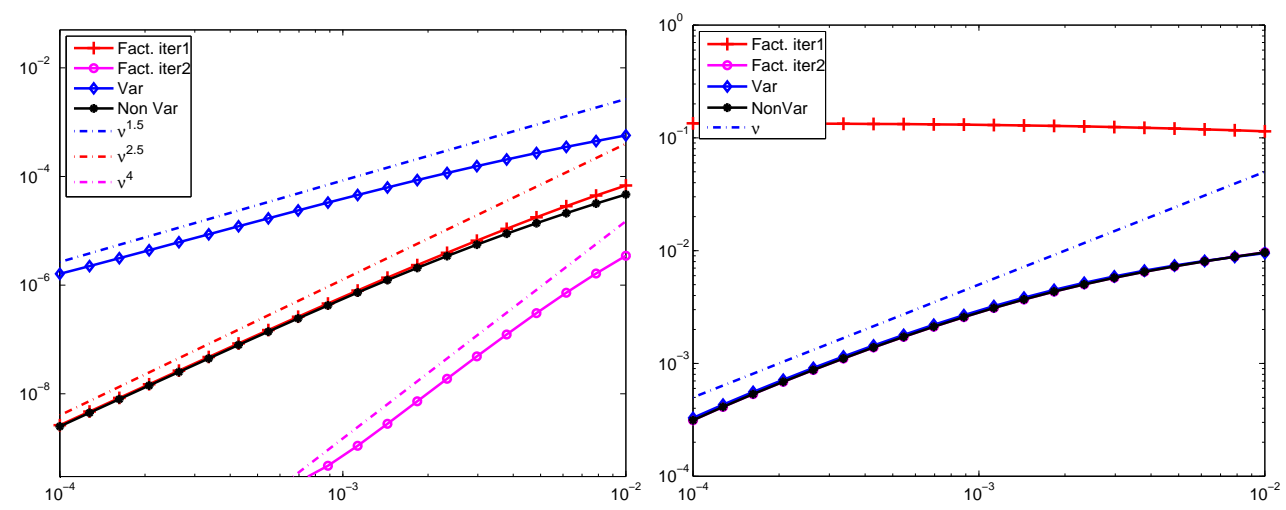

FIG. 5.3. Errors for $a>0$ as the viscosity becomes small for our factorization algorithm compared to other coupling algorithms from the literature. Left: $\left\|u-u_{a d}\right\|_{L_{x, t}^{2}}$. Right: $\left\|u-u_{a}\right\|_{L_{x, t}^{2}}$
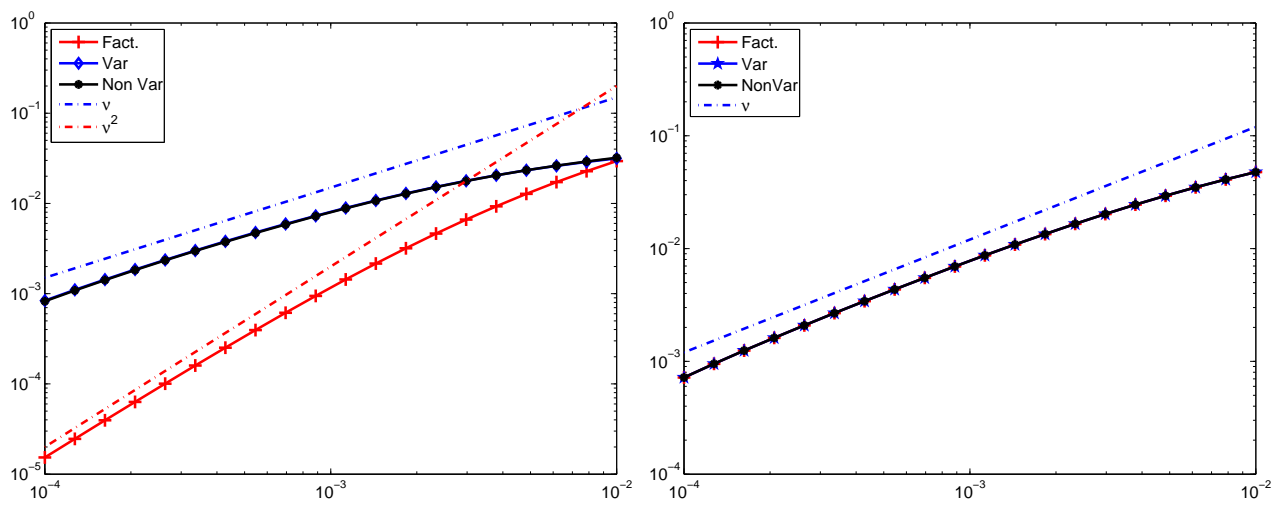

FIG. 5.4. Errors for $a<0$ as the viscosity becomes small for the factorization algorithm compared to other coupling algorithms from the literature. Left: $\left\|u-u_{a d}\right\|_{L_{x, t}^{2}}$. Right: $\left\|u-u_{a}\right\|_{L_{x, t}^{2}}$

5.2. Negative advection. We now consider a negative advection example, $a=$ -1 , with initial condition

$$
u_{0}(x)=e^{-100\left(x-x_{0}\right)^{2}}, \text { with } x_{0}=0.5 .
$$

Figure 5.4 shows the $L^{2}$ space-time error between the viscous solution and the solution of the factorization algorithm (2.6), and also a comparison to the errors of the other coupling algorithms from the literature; the variational coupling conditions for $a<0$ are $-\nu \partial_{x} u_{a d}(0, \cdot)+a u_{a d}(0, \cdot)=a u_{a}(0, \cdot)$, and the non-variational ones are $u_{a d}(0, \cdot)=$ $u_{a}(0, \cdot)$. Once again the error in $\Omega_{2}$ is $\mathcal{O}(\nu)$ for each algorithm, since each algorithm solves the same advection equation in $\Omega_{2}$. However the factorization algorithm solves then a second advection equation which provides a better boundary value for the advection-diffusion problem in $\Omega_{1}$ and thus can provide an error $\mathcal{O}\left(\nu^{2}\right)$, whereas the other algorithms only give an approximation $\mathcal{O}(\nu)$ in $\Omega_{1}$.

6. Conclusions. We introduced a new algorithm to solve advection diffusion problems with pure advection approximation in a subregion. We call this algorithm factorization algorithm, because it is based on a factorization of the underlying operator. We proved rigorous error estimates that show that our new algorithm gives 
solutions that are closer to the fully viscous solution of interest than other coupling algorithms in the literature. Our numerical experiments indicate that our estimates are sharp, an issue we are currently investigating using multiscale expansions.

\section{REFERENCES}

[1] V. Agoshkov, P. Gervasio, and A. Quarteroni, Optimal control in heterogeneous domain decomposition methods for advection-diffusion equations, Mediterr. J. Math., 3 (2006), pp. 147-176.

[2] A. Bamberger, B. Engquist, L. Halpern, and P. Joly, Higher order paraxial wave equation approximations in heterogeneous media, SIAM J. Appl. Math., 48 (1988), pp. 129-154.

[3] L. Barbu, C. Coclici, And G. Morosanu, A class of singularly perturbed, coupled, evolutionary boundary value problems, Math. Sci. Res. Hot-Line, 4 (2000), pp. 63-76.

[4] D. Bennequin, M. J. Gander, and L. Halpern, A homographic best approximation problem with application to optimized Schwarz waveform relaxation, Math. Comp., 78 (2009), pp. 185-223.

[5] F. Brezzi, C. Canuto, And A. Russo, A self-adaptive formulation for the Euler/Navier-Stokes coupling, Comput. Methods Appl. Mech. Engrg., 73 (1989), pp. 317-330.

[6] C. A. Coclici, G. Moroşanu, and W. L. Wendland, The coupling of hyperbolic and elliptic boundary value problems with variable coefficients, Math. Methods Appl. Sci., 23 (2000), pp. 401-440.

[7] C. A. COCLICI AND W. L. WENDLAND, Analysis of a heterogeneous domain decomposition for compressible viscous flow, Mathematical Models and Methods in Applied Sciences, 11 (2001), pp. 565-599.

[8] C. A. Coclici, W. L. Wendland, J. Heiermann, and M. Auweter-Kurtz, A heterogeneous domain decomposition for initial-boundary value problems with conservation laws and electromagnetic fields, in Twelfth International Conference on Domain Decomposition Methods, Chiba, Japan, T. Chan, T. Kako, H. Kawarada, and O. Pironneau, eds., Bergen, 2001, Domain Decomposition Press, pp. 281-288.

[9] S. Deparis, M. Discacciati, G. Fourestey, and A. Quarteroni, Heterogeneous domain decomposition methods for fluid-structure interaction problems, in Domain Decomposition Methods in Science and Engineering XVI, Springer, 2007, pp. 41-52.

[10] Q. V. Dinh, R. Glowinski, J. PÉriaux, and G. Terrasson, On the coupling of viscous and inviscid models for incompressible fluid flows via domain decomposition, in First International Symposium on Domain Decomposition Methods for Partial Differential Equations (Paris, 1987), SIAM, Philadelphia, PA, 1988, pp. 350-369.

[11] - On the coupling of viscous and inviscid models for incompressible fluid flows via domain decomposition, in First International Symposium on Domain Decomposition Methods for Partial Differential Equations (Paris, 1987), SIAM, Philadelphia, PA, 1988, pp. 350-369.

[12] M. Discacciati, . Gervasio P, AND A. QuARTERoni, "heterogeneous mathematical models in fluid dynamics and associated solution algorithms.", Tech. Report MOX 04/2010, (2010).

[13] E. DuBACH, Contribution à la Résolution des Équations fluides en domaine non borné, PhD thesis, Université Paris 13, 1993.

[14] B. EngQuist AND L. YING, Sweeping preconditioner for the helmholtz equation: moving perfectly matched layers, Multiscale Modeling \& Simulation, 9 (2011), pp. 686-710.

[15] L. Formaggia, J.-F. Gerbeau, F. Nobile, And A. Quarteroni, On the coupling of $3 d$ and $1 d$ navier-stokes equations for flow problems in compliant vessels, Computer Methods in Applied Mechanics and Engineering, 191 (2001), pp. 561-582.

[16] L. Formaggia, A. Quarteroni, And A. Veneziani, Cardiovascular Mathematics: Modeling and simulation of the circulatory system, vol. 1, Springer, 2009.

[17] M. J. Gander, Optimized Schwarz methods, SIAM J. Numer. Anal., 44 (2006), pp. 699-731.

[18] M. J. Gander, L. Halpern, And C. JAPHet, Optimized Schwarz algorithms for coupling convection and convection-diffusion problems, in Proceedings of the 13th international conference of Domain Decomposition, 2001, pp. 253-260.

[19] M. J. Gander, L. Halpern, C. Japhet, And V. Martin, Advection diffusion problems with pure advection approximation in subregions, in Domain Decomposition Methods in Science and Engineering XVI, O. B. Widlund and D. E. Keyes, eds., vol. XVI of Lecture Notes in Computational Science and Engineering 55, Springer-Verlag, 2007, pp. 239-246.

[20] M. J. Gander, L. Halpern, C. JAphet, And V. Martin, Viscous problems with inviscid approximations in subregions: a new approach based on operator factorization, in CANUM 2008, vol. 27 of ESAIM Proc., EDP Sci., Les Ulis, 2009, pp. 272-288. 
[21] M. J. GANDER AND F. NATAF, AILU: a preconditioner based on the analytic factorization of the elliptic operator, Numerical linear algebra with applications, 7 (2000), pp. 543-567.

[22] M. J. Gander AND F. NATAF, An incomplete lu preconditioner for problems in acoustics, Journal of Computational Acoustics, 13 (2005), pp. 455-476.

[23] F. Gastaldi and A. Quarteroni, On the coupling of hyperbolic and parabolic systems: analytical and numerical approach, Appl. Numer. Math., 6 (1989/90), pp. 3-31. Spectral multi-domain methods (Paris, 1988).

[24] F. Gastaldi, A. Quarteroni, and G. S. Landriani, On the coupling of two dimensional hyperbolic and elliptic equations : Analytical and numerical approach, in Third International Symposium on Domain Decomposition Methods for Partial Differential Equations, T. C. et al eds., ed., Philadelphia, 1990, SIAM, pp. 22-63.

[25] P. Gervasio, J.-L. Lions, And A. Quarteroni, Heterogeneous coupling by virtual control methods, Numer. Math., 90 (2001), pp. 241-264.

[26] L. Halpern, Artificial boundary conditions for the linear advection diffusion equation, Mathematics of computation, 46 (1986), pp. 425-438.

[27] C.-H. Lai, A. M. Cuffe, AND K. A. Pericleous, A defect equation approach for the coupling of subdomains in domain decomposition methods, Comput. Math. Appl., 35 (1998), pp. 8194.

[28] J.-L. Lions and E. Magenes, Problèmes aux limites non homogènes et applications, vol. 17-18 of Travaux et recherches mathématiques, Dunod, 1968.

[29] J.-P. LohÉac, F. Nataf, and M. Schatzman, Parabolic approximations of the convectiondiffusion equation, Math. Comp., 60 (1993), pp. 515-530.

[30] V. Martin, An optimized Schwarz waveform relaxation method for unsteady convection diffusion equation, Appl. Numer. Math., 52 (2005), pp. 401-428.

[31] G. MÉTIVIER, Small viscosity and boundary layer methods, Modeling and Simulation in Science, Engineering and Technology, Birkhäuser Boston Inc., Boston, MA, 2004. Theory, stability analysis, and applications.

[32] R. Mittal and G. Iaccarino, Immersed boundary methods, Annu. Rev. Fluid Mech., 37 (2005), pp. 239-261.

[33] H. J.-P. Morand And R. Ohayon, Fluid Structure Interaction, John Wiley \& Sons, 1995.

[34] F. NATAF, Résolution de l'équation de convection-diffusion stationnaire par une factorisation parabolique, C. R. Acad. Sci. Paris Sér. I Math., 310 (1990), pp. 869-872.

[35] F. NATAF AND F. Rogier, Factorization of the convection-diffusion operator and the Schwarz algorithm, Math. Models Methods Appl. Sci., 5 (1995), pp. 67-93.

[36] A. Quarteroni, F. Pasquarelli, and A. Valli, Heterogeneous domain decomposition: principles, algorithms, applications, in Fifth International Symposium on Domain Decomposition Methods for Partial Differential Equations (Norfolk, VA, 1991), Siam Philadelphia, PA, 1992, pp. 129-150. 